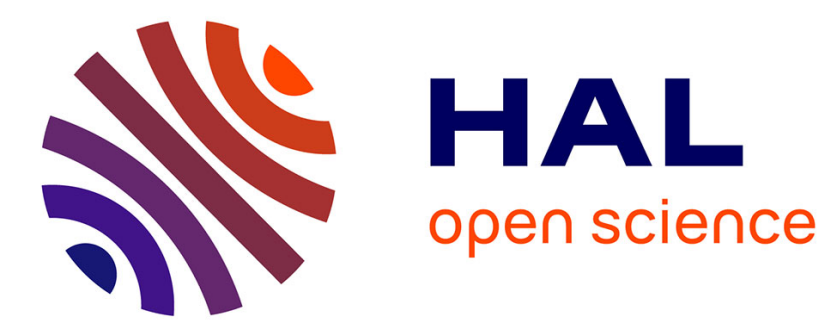

\title{
TENSOR RANK: MATCHING POLYNOMIALS AND SCHUR RINGS
}

Dima Grigoriev, Mikhail Muzychuk, Ilya Ponomarenko

\section{To cite this version:}

Dima Grigoriev, Mikhail Muzychuk, Ilya Ponomarenko. TENSOR RANK: MATCHING POLYNOMIALS AND SCHUR RINGS. Foundations of Computational Mathematics, 2014. hal-03044846

\section{HAL Id: hal-03044846 \\ https://hal.science/hal-03044846}

Submitted on 7 Dec 2020

HAL is a multi-disciplinary open access archive for the deposit and dissemination of scientific research documents, whether they are published or not. The documents may come from teaching and research institutions in France or abroad, or from public or private research centers.
L'archive ouverte pluridisciplinaire HAL, est destinée au dépôt et à la diffusion de documents scientifiques de niveau recherche, publiés ou non, émanant des établissements d'enseignement et de recherche français ou étrangers, des laboratoires publics ou privés. 


\title{
TENSOR RANK: MATCHING POLYNOMIALS AND SCHUR RINGS
}

\author{
DIMA GRIGORIEV, MIKHAIL MUZYCHUK, AND ILYA PONOMARENKO
}

\begin{abstract}
We study the polynomial equations vanishing on tensors of a given rank. By means of polarization we reduce them to elements $A$ of the group algebra $\mathbb{Q}\left[S_{n} \times S_{n}\right]$ and describe explicit linear equations on the coefficients of $A$ to vanish on tensors of a given rank. Further, we reduce the study to the Schur ring over the group $S_{n} \times S_{n}$ that arises from the diagonal conjugacy action of $S_{n}$. More closely, we consider elements of $\mathbb{Q}\left[S_{n} \times S_{n}\right]$ vanishing on tensor of rank $n-1$ and describe them in terms of triples of Young diagrams, their irreducible characters and nonvanishing of their Kronecker coefficients. Also, we construct a family of elements in $\mathbb{Q}\left[S_{n} \times S_{n}\right]$ vanishing on tensors of rank $n-1$ and illustrate our approach by a sharp lower bound on the border rank of an explicitly produced tensor. Finally, we apply this construction to prove a lower bound $5 n^{2} / 4$ on the border rank of the matrix multiplication tensor (being, of course, weaker than the best known one $(2-\epsilon) \cdot n^{2}$, due to Landsberg, Ottaviani).
\end{abstract}

\section{INTRODUCTION}

In this paper we propose an approach to producing equations for tensors of a given rank with the goal of obtaining lower bounds on the rank. We recall (see e.g. $[22,25,4,12,13])$ that the $\operatorname{rank} \operatorname{rk}(A)$ of a tensor $A \in U \otimes V \otimes W$ is defined to be the minimal positive integer $r$ such that there exist vectors $u^{(i)} \in U, v^{(i)} \in V$, $w^{(i)} \in W, i=1, \ldots, r$, for which

$$
A=\sum_{1 \leq i \leq r} u^{(i)} \otimes v^{(i)} \otimes w^{(i)} .
$$

(Throughout this paper we assume that the vector spaces $U, V$ and $W$ are defined over an algebraically closed field of characteristic zero.) Clearly, the concept of the rank of a tensor generalizes the one of the matrix rank. But unlike the matrix rank the tensor rank is not semicontinuous. That is why one studies the border rank $\underline{\mathrm{rk}}(A)$ being the maximal semicontinuous function for which $\underline{\mathrm{rk}}(A) \leq \mathrm{rk}(A)$.

The tensor rank equals the multiplicative complexity of computing a family of bilinear forms [22]. One of the main inspiring problems in this context is to estimate the multiplicative complexity of $n \times n$ matrix multiplication, that is equal to the rank rk $\left(M_{n}\right)$ of the structure tensor $M_{n}$ of the algebra of $n \times n$ matrices. The best known bounds are

$$
2.5 \cdot n^{2}-3 n \leq \operatorname{rk}\left(M_{n}\right) \leq O\left(n^{2.38}\right),
$$

we refer to $[25,4,12,13]$ for the development and the history of the upper bound, and to [2] for the lower bound. In [19] it was established a lower bound on the border rank $\underline{r k}\left(M_{n}\right) \geq 3 n^{2} / 2+n / 2-1$. In [17] the best known current bound $\underline{\mathrm{rk}}\left(M_{n}\right) \geq(2-\epsilon) \cdot n^{2}$ for any $\epsilon>0$ was proved. 
Thus, the gap between the upper and lower bounds is big. One of its reasons is the lack of explicit equations on the variety $\mathcal{T}_{r} \subset U \otimes V \otimes W$ of the tensors with the border rank less or equal to $r$. There are several approaches in this direction.

Strassen in [23] has constructed explicit equations on the variety $\mathcal{T}_{r}$ for certain $r$ 's in the case $\operatorname{dim}(U)=3$ and $\operatorname{dim}(V)=\operatorname{dim}(W)=n$. This result was extended in [15] to more general tensors of order more than 3 . Another approach is based on the general idea of "embedding" tensors into appropriate matrices (called flattenings) and estimating the rank of these matrices [16, 14, 7, 17]. A study of the closures of the $\mathrm{GL}(U) \times \mathrm{GL}(V) \times \mathrm{GL}(W)$-orbits of tensors was proposed first in [24] being developed further in [5], the recent progress in [6] has allowed one to obtain a bound close to [19], relying on this study.

A similar problem of estimating the rank for the symmetric product (rather than the tensor product) was studied e.g. in [18] (see also the numerous references in the latter paper); earlier a method to obtain lower bound for the rank in this situation was suggested in [8]. We mention also a topological approach that was proposed in [9] for a related problem on lower bounds for the complexity of polynomials.

Let us briefly discuss the contents of our paper. In Section 2 we establish a reduction from general polynomials on tensors $A=\left(A_{i j k}\right)$ to the matching polynomials which are homogeneous, and polylinear in a strong sense: the indices of variables $X_{i j k}$ occuring in any given monomial form a 3-dimensional matching

$$
\left\{\left(i^{f}, i^{g}, i^{h}\right): i=1, \ldots, n\right\}
$$

where $n$ is the degree of the matching polynomial, and $f, g, h \in S_{n}$ are permutations depending on the monomial. One can treat such a matching polynomial on $n \times n \times n$ tensors, which vanishes on the rank $n-1$ tensors, as a 3 -dimensional analogue of the customary determinant (or more generally, a 3-dimensional subdeterminant vanishing on tensors of fixed rank $r<n)$. Our reduction is a special polarization which preserves the property "to vanish on $\mathcal{T}_{r}$ ". Subsequently, having a 3-dimensional determinant $D$ one can pass to a polynomial vanishing on $\mathcal{T}_{r}$ for tensors of a smaller size $n_{1} \times n_{2} \times n_{3}\left(n_{1}, n_{2}, n_{3} \leq n\right)$ by means of depolarization just identifying suitable variables of $D$. Since the polarization and the depolarization are transformation being inverse to each other, one may reduce the study of equations on $\mathcal{T}_{r}$ to matching polynomials.

In its turn the 3 -dimensional matchings (1) are in 1-1 correspondence with the elements $\left(f^{-1} g, f^{-1} h\right)$ of the group $S_{n} \times S_{n}$. This enables us to identify a matching polynomial with an element of the group algebra $\mathbb{Q}\left[S_{n} \times S_{n}\right]$. In Section 3 we describe explicitly (linear) equations on the coefficients of an element of this algebra that corresponds to a 3-dimensional (sub)determinant vanishing on tensors of rank at most $r$. Since these equations, and thereby, their space of solutions

$$
V_{n, n-r} \subset \mathbb{Q}\left[S_{n} \times S_{n}\right]
$$

are invariant under the (diagonal) conjugacy action of $S_{n}$, the space $V_{n, n-r}$ is generated as a right ideal in $\mathbb{Q}\left[S_{n} \times S_{n}\right]$ by the intersection $V_{n, n-r} \cap \mathcal{A}$ where $\mathcal{A} \subset \mathbb{Q}\left[S_{n} \times S_{n}\right]$ is the Schur ring of this action (see Section 4). Moreover,

$$
V_{n, n-r} \cap \mathcal{A}=\bigoplus_{\pi} V_{n, n-r} \cap \mathcal{A}_{\pi}
$$


where the direct sum ranges over the irreducible representations of $\mathcal{A}$. Furthermore, in Section 4 we describe the latter representations in terms of triples of Young diagrams and nonvanishing of their Kronecker coefficients. Finally, in this section we provide the conditions (in terms of the Young diagrams) when the depolarization does not vanish identically.

In Section 5 we study more closely the case of the rank of $n \times n \times n$ tensors equal $n-1$, and give an explicit criterion for a matching polynomial to be a 3 dimensional determinant; this criterion is expressed in terms of the triples of Young diagrams. Also in this section we show that, unfortunately, if a depolarization of 3 -dimensional determinant does not vanish on $n_{1} \times n_{2} \times n_{3}$ tensors, then $n_{i}>n / 3$ for some $i \in\{1,2,3\}$. This implies that in order to obtain nonlinear lower bound on the tensor rank one should consider elements of $V_{n, n-r}$ with $r<n-1$ (perhaps, with the rank $r$ significantly less than $n-1$ ).

Finally, in Section 5 we construct a particular family of elements in $V_{n, n-1}$ which we apply in Section 6 to yield a $(2 m+1) \times(2 m+1) \times(2 m+1)$ tensor $A$ such that $\mathrm{rk}(A)=\mathrm{rk}(A)=3 \mathrm{~m}$. Also as an illustration of our approach we apply in Section 6 the latter construction to get a bound $\underline{\mathrm{rk}}\left(M_{n}\right) \geq 5 n^{2} / 4$ (being, of course, weaker than the best known bound from [19]).

\section{Notations.}

For positive integers $m \leq n$ we set $[m, n]=\{m, m+1, \ldots, n\}$ and $[n]=[1, n]$.

Given a right action of a group $G$ on a set $\Omega$, we write $\omega^{g}$ for an image of $\omega \in \Omega$ under an action of $g \in G$.

The set of all (resp. $r$-class) partitions of $[n]$ is denoted by $\Lambda(n)$ (resp. $\Lambda(n, r)$ ). For the sake of convenience we agree that partitions can contain empty subsets (in particular, $r$ can be greater than $n$ ). In other words, a partition $\lambda \in \Lambda(n, r)$ is treated as a map $\lambda:[n] \rightarrow[r]$. To make our notation consistent, we write $x^{\lambda}$ instead of $\lambda(x)$.

The Young diagram of $\lambda \in \Lambda(n)$ is denoted by [ $\lambda]$. Clearly, empty subsets from a partition do not influence on the Young diagram. The set of all Young diagrams with $n$ nodes is denoted by $\boldsymbol{\Lambda}(n)$. The Young subgroup of a partition $\lambda \in \Lambda(n)$ is denoted by $S_{\lambda}$. Notice that $S_{\lambda}=\left\{g \in S_{n} \mid g \lambda=\lambda\right\}$.

Given a group $G$ and a set $H \subset G$ the sum $\sum_{h \in H} h$ in the group algebra $\mathbb{Q} G=\mathbb{Q} G$ is denoted by $\underline{H}$. If $H$ is a subgroup of $G$, then $\frac{1}{|H|} \underline{H}$ is an idempotent of the group algebra $\mathbb{Q} G$ denoted by $e_{H}$. Recall that $H \leq G$ means that $H$ is a subgroup of $G$. The product of two elements $x, y \in \mathbb{Q} G$ is written either as $x y$ or $x \cdot y$. The identity of a group $G$ is denoted by $1_{G}$.

Inside a group $G=S_{n}^{3}$ we fix two subgroups: $D=\left\{(d, d, d) \mid d \in S_{n}\right\}$ and $S=\left\{\left(1, g_{2}, g_{3}\right) \mid g_{2}, g_{3} \in S_{n}\right\}$. Notice that $S$ is normal in $G$ and $G=S \rtimes D$. The subgroup $S$ is isomorphic to $S_{n}^{2}$ and we denote by $\iota: S_{n}^{2} \rightarrow S$ the natural embedding $\iota\left(\left(g_{2}, g_{3}\right)\right)=\left(1, g_{2}, g_{3}\right)$.

Given three elements $x^{i}=\sum_{g \in S_{n}} x_{g}^{i} g$ of a group algebra $\mathbb{Q} S_{n}$, we denote by $x^{1} \otimes x^{2} \otimes x^{3}$ the element $\sum_{g_{1} \in S_{n}, g_{2} \in S_{n}, g_{3} \in S_{n}} x_{g_{1}}^{1} x_{g_{2}}^{2} x_{g_{3}}^{3}\left(g_{1}, g_{2}, g_{3}\right) \in \mathbb{Q} S_{n}^{3}$.

For a set $S$ the algebra of all rational $S \times S$-matrices is denoted by $\operatorname{Mat}_{S}(\mathbb{Q})$, or $\operatorname{Mat}_{n}(\mathbb{Q})$ if $S=[n]$. 


\section{TENSOR RANK, POLARIZATION AND MATCHING POLYNOMIALS}

We observe that the variety $\mathcal{T}_{r}$ (see Section 1 ) is defined over the field $\mathbb{Q}$. Therefore it suffices to look for polynomials which vanish on $\mathcal{T}_{r}$ with rational coefficients. Thus throughout the paper we assume that all the polynomials have rational coefficients.

Let $\mathcal{P}\left(n_{1}, n_{2}, n_{3}\right)$ be the set of all homogeneous polynomials $P(X)$ on $n_{1} \times n_{2} \times$ $n_{3}$ tensors; here $X=\left\{X_{i j k}\right\}_{i \in\left[n_{1}\right], j \in\left[n_{2}\right], k \in\left[n_{3}\right]}$ denotes the set of variables. Our goal is to find those polynomials $P(X)$ for which $P(A)=0$ for all $n_{1} \times n_{2} \times n_{3}$ tensors $A$ of rank at most $r$. The linear space of all these polynomials is denoted by $\mathcal{P}_{r}\left(n_{1}, n_{2}, n_{3}\right)$. The key point of our approach to look for elements of this space is the concept of a matching polynomial introduced below.

Given an arbitrary triple $g=\left(g_{1}, g_{2}, g_{3}\right)$ of functions $g_{i}:[n] \rightarrow\left[n_{i}\right], i=1,2,3$ we define

$$
M_{g}(X)=\prod_{i=1}^{n} X_{i^{g_{1}, i^{g_{2}}, i^{g_{3}}} .}
$$

In the case when all $g_{i}$ 's are permutations of $[n]$ we call the above product a matching monomial. Notice that different triples may define the same monomial. More precisely, we have the following

Lemma 2.1. Given two triples $g=\left(g_{1}, g_{2}, g_{3}\right)$ and $\left(h_{1}, h_{2}, h_{3}\right)$ of functions, $g_{i}, h_{i}$ : $[n] \rightarrow\left[n_{i}\right]$, two monomials $M_{g}(X)$ and $M_{h}(X)$ are equal if and only if there exists a permutation $f \in S_{n}$ such that $f g_{i}=h_{i}, i=1,2,3$.

Proof. The "if" direction is trivial. Assume now that $M_{g}(X)=M_{h}(X)$. Then the multisets $\left\{\left(i^{g_{1}}, i^{g_{2}}, i^{g_{3}}\right) \mid i \in[n]\right\}$ and $\left\{\left(i^{h_{1}}, i^{h_{2}}, i^{h_{3}}\right) \mid i \in[n]\right\}$ are equal, that is there exists a bijection between the triples hereby producing the required $f \in S_{n}$..

It follows from the above statement that matching monomials are in one-to-one correspondence with the triples $g=\left(1, g_{2}, g_{3}\right), g_{2}, g_{3} \in S_{n}$ :

$$
M_{g}(X)=\prod_{i=1}^{n} X_{i i^{g_{2}} i^{g_{3}}},
$$

In what follows we will also abbreviate $M_{\iota(g)}(X)$ as $M_{g}(X)$ where $g \in S_{n}^{2}$. We say that $P(X) \in \mathcal{P}(n, n, n)$ is a (3-dimensional) matching polynomial if it is a linear combination of matching monomials The linear space of matching polynomials is denoted by $\mathcal{M}(n)$.

Given a monomial $M(X)=\prod_{i j k} X_{i j k}^{m_{i j k}} \in \mathcal{P}\left(n_{1}, n_{2}, n_{3}\right)$, define $u \in \mathbb{Z}^{n_{1}}, v \in \mathbb{Z}^{n_{2}}$ and $w \in \mathbb{Z}^{n_{3}}$ as follows $u_{s}=\sum_{j k} m_{s j k}, v_{s}=\sum_{i k} m_{i s k}, w_{s}=\sum_{i j} m_{i j s}$. The triple $(u, v, w) \in \mathbb{Z}^{n_{1}} \times \mathbb{Z}^{n_{2}} \times \mathbb{Z}^{n_{3}}$ will be called the multidegree of $M(X)$. Notice that we always have that $\sum_{i} u_{i}=\sum_{j} v_{j}=\sum_{k} w_{k}$ (it is the degree of $M(X)$ ). The sum of all monomials of a polynomial $P(X) \in \mathcal{P}\left(n_{1}, n_{2}, n_{3}\right)$ that have the same multidegree is called a multihomogeneous component of $P(X)$. The polynomial $P(X) \in \mathcal{P}\left(n_{1}, n_{2}, n_{3}\right)$ is multihomogeneous if it has exactly one multihomogeneous component, $(u, v, w)$; the polynomial $P(X)$ is also called $N$-uniform where $N=$ $\sum_{i} u_{i}$

Polarization. Given an $N$-uniform polynomial $P(X) \in \mathcal{P}\left(n_{1}, n_{2}, n_{3}\right)$ one can construct a matching polynomial belonging to $\mathcal{M}(N)$ by means of its polarization. 
Since the polarization is a linear operator it suffices to define it on a monomial $M(X)=\prod_{i j k} X_{i j k}^{m_{i j k}} \in \mathcal{P}\left(n_{1}, n_{2}, n_{3}\right)$.

Consider three partitions $\mu \in \Lambda\left(N, n_{1}\right), \nu \in \Lambda\left(N, n_{2}\right), \kappa \in \Lambda\left(N, n_{3}\right)$ with the cardinalities of their classes $u, v, w$, respectively. Rewrite $M(X)$ as a product (in an arbitrary order) of $N$ variables $X_{i j k}$ repeating the latter $m_{i j k}$ times. Replace in this product $u_{i}$ occurances of subscript $i$ by different elements of $i$-th class of $\mu$ (in an arbitrary way); similar for $v_{j}$ occurances of $j$ and $\nu$; and for $w_{k}$ and $\kappa$, respectively. The resulting matching monomial denote by $\bar{M} \in \mathcal{M}(N)$. The polarization of $M(X)$ is defined as

$$
\frac{1}{\prod_{1 \leq i \leq n_{1}} u_{i} ! \cdot \prod_{1 \leq j \leq n_{2}} v_{j} ! \cdot \prod_{1 \leq k \leq n_{3}} w_{k} !} \sum_{g_{1} \in S_{\mu}, g_{2} \in S_{\nu}, g_{3} \in S_{\kappa}} \bar{M}_{g_{1}, g_{2}, g_{3}} \in \mathcal{M}(N)
$$

Example. The polarization of monomial $X_{211} X_{121} X_{112}$ equals

$\frac{1}{8}\left(X_{211} X_{123} X_{332}+X_{211} X_{323} X_{132}+X_{231} X_{123} X_{312}+X_{231} X_{323} X_{112}+X_{213} X_{121} X_{332}+\right.$ $\left.X_{213} X_{321} X_{132}+X_{233} X_{121} X_{312}+X_{233} X_{321} X_{112}\right)$

Lemma 2.2. Let $P(X) \in \mathcal{P}_{r}\left(n_{1}, n_{2}, n_{3}\right)$. Then

(1) each multihomogeneous component of $P(X)$ belongs to $\mathcal{P}_{r}\left(n_{1}, n_{2}, n_{3}\right)$,

(2) if $P(X)$ is $N$-uniform, then the polarization of $P(X)$ belongs to $\mathcal{P}_{r}(N, N, N)$.

Proof. To prove statement (1) take $n_{1}+n_{2}+n_{3}$ new variables $\varepsilon_{s_{u}}^{(u)}$ with $s_{u} \in\left[n_{u}\right]$ and $u \in[3]$. Let us consider

$$
P\left(\ldots, X_{i j k}:=X_{i j k} \varepsilon_{i}^{(1)} \varepsilon_{j}^{(2)} \varepsilon_{k}^{(3)}, \ldots\right) .
$$

as a polynomial in these variables. The coefficient of this polynomial at the monomial $M=\prod_{u, s_{u}}\left(\varepsilon_{s_{u}}^{(u)}\right)^{d\left(s_{u}, u\right)}$ coincides with the multihomogeneous component of $P(X)$, the multidegree of which is $\left(\ldots, d\left(s_{u}, u\right), \ldots\right)$ where $d\left(s_{u}, u\right)$ is the degree of the variable $\varepsilon_{s_{u}}^{(u)}$ in $M$. Now, since $P(X) \in \mathcal{P}_{r}\left(n_{1}, n_{2}, n_{3}\right)$, the above coefficient at $M$ is equal to zero. This implies that the multihomogeneous component of $P(X)$ that contains $M$, vanishes at any $n_{1} \times n_{2} \times n_{3}$ tensor of rank $\leq r$. Thus each multihomogeneous component of a polynomial $P(X)$ belongs to $\mathcal{P}_{r}\left(n_{1}, n_{2}, n_{3}\right)$.

To prove statement (2) denote by $Q(Y) \in \mathcal{M}(N)$ the polarization of $P(X)$ (see (4)). Thus, $P(X)$ is defined on $n_{1} \times n_{2} \times n_{3}$ tensors for some integers $n_{1}, n_{2}, n_{3}$, while $Q(Y)$ is defined on $N \times N \times N$ tensors.

Take any $N \times N \times N$ tensor $A$ of rank $\leq r$. Then there exist $N$-dimensional vectors $X^{(u)}, Y^{(u)}, Z^{(u)}, 1 \leq u \leq r$ such that

$$
A_{\alpha, \beta, \gamma}=\sum_{u=1}^{r} X_{\alpha}^{(u)} Y_{\beta}^{(u)} Z_{\gamma}^{(u)}
$$

for all $\alpha, \beta, \gamma$. Denote by $\mu_{1}, \ldots, \mu_{n_{1}} \subseteq[N]$ the classes of partition $\mu$, respectively by $\nu_{1}, \ldots, \nu_{n_{2}} \subseteq[N]$ the classes of partition $\nu$, and by $\kappa_{1}, \ldots, \kappa_{n_{3}} \subseteq[N]$ the classes of partition $\kappa$ (see (4)).

For non-empty subsets $I_{1} \subseteq \mu_{1}, \ldots, I_{n_{1}} \subseteq \mu_{n_{1}}$ denote by $X^{(u)}\left(I_{1}, \ldots, I_{n_{1}}\right)$ a vector of dimension $n_{1}$ with $i$-th coordinate equal $\sum_{\alpha \in I_{i}} X_{\alpha}^{(u)}, 1 \leq i \leq n_{1}$. In a similar way, for non-empty subsets $J_{1} \subseteq \nu_{1}, \ldots, J_{n_{2}} \subseteq \nu_{n_{2}}$ we define $n_{2}$-dimensional vector $Y^{(u)}\left(J_{1}, \ldots, J_{n_{2}}\right)$, and for non-empty subsets $K_{1} \subseteq \kappa_{1}, \ldots, K_{n_{3}} \subseteq \kappa_{n_{3}}$ we define $n_{3}$-dimensional vector $Z^{(u)}\left(K_{1}, \ldots, K_{n_{3}}\right)$. Consider $n_{1} \times n_{2} \times n_{3}$ tensor 


$$
\begin{aligned}
& A\left(I_{1}, \ldots, I_{n_{1}} ; J_{1}, \ldots, J_{n_{2}} ; K_{1}, \ldots, K_{n_{3}}\right):= \\
& \sum_{1 \leq u \leq r} X^{(u)}\left(I_{1}, \ldots, I_{n_{1}}\right) \otimes Y^{(u)}\left(J_{1}, \ldots, J_{n_{2}}\right) \otimes Z^{(u)}\left(K_{1}, \ldots, K_{n_{3}}\right) \text { (cf. (5)). Then } \\
& Q(A)=\frac{1}{\prod_{1 \leq i \leq n_{1}} u_{i} ! \cdot \prod_{1 \leq j \leq n_{2}} v_{j} ! \cdot \prod_{1 \leq k \leq n_{3}} w_{k} !} . \\
& \sum_{I_{i} \subseteq \mu_{i}, 1 \leq i \leq n_{1} ; J_{j} \subseteq \nu_{j}, 1 \leq j \leq n_{2} ; K_{k} \subseteq \kappa_{k}, 1 \leq k \leq n_{3}} P\left(A\left(I_{1}, \ldots, I_{n_{1}} ; J_{1}, \ldots, J_{n_{2}} ; K_{1}, \ldots, K_{n_{3}}\right)\right)
\end{aligned}
$$

where the summation ranges over non-empty subsets $I_{1}, \ldots, I_{n_{1}} ; J_{1}, \ldots, J_{n_{2}} ; K_{1}, \ldots, K_{n_{3}}$. Since obviously $\operatorname{rk}\left(A\left(I_{1}, \ldots, I_{n_{1}} ; J_{1}, \ldots, J_{n_{2}} ; K_{1}, \ldots, K_{n_{3}}\right)\right) \leq r$ for all subsets $I_{1}, \ldots, I_{n_{1}}$; $J_{1}, \ldots, J_{n_{2}} ; K_{1}, \ldots, K_{n_{3}}$ and $P(X) \in \mathcal{P}_{r}\left(n_{1}, n_{2}, n_{3}\right)$ the right-hand side of (6) vanishes, thus we are done.

Depolarization. Given three functions $\lambda_{i}:[N] \rightarrow\left[n_{i}\right], i=1,2,3$, one obtains an algebra epimorphism called $\left(\lambda_{1}, \lambda_{2}, \lambda_{3}\right)$-contraction, from $\mathbb{C}\left[X_{i j k}\right]_{i \in[N], j \in[N], k \in[N]}$ onto $\mathbb{C}\left[Y_{i j k}\right]_{i \in\left[n_{1}\right], j \in\left[n_{2}\right], k \in\left[n_{3}\right]}$ via $\left(X_{i j k}\right)^{\lambda_{1}, \lambda_{2} \lambda_{3}}=Y_{i^{\lambda_{1}} j^{\lambda_{2}} k^{\lambda_{3}}}$. The image of a polynomial $P(X) \in \mathcal{M}(N)$ will be denoted as $P^{\lambda_{1}, \lambda_{2}, \lambda_{3}}(Y)$. Thus, $P^{\lambda_{1}, \lambda_{2}, \lambda_{3}}(Y) \in$ $\mathcal{P}\left(n_{1}, n_{2}, n_{3}\right)$.

Lemma 2.3. Let $g=\left(g_{1}, g_{2}, g_{3}\right), h=\left(h_{1}, h_{2}, h_{3}\right) \in S_{N}^{3}$ be arbitrary elements. Then

$$
\left(M_{g}(X)\right)^{\lambda_{1}, \lambda_{2} \lambda_{3}}=\left(M_{h}(X)\right)^{\lambda_{1}, \lambda_{2} \lambda_{3}} \Longleftrightarrow D g S_{\lambda}=D h S_{\lambda}
$$

where $S_{\lambda}=S_{\lambda_{1}} \times S_{\lambda_{2}} \times S_{\lambda_{3}}$.

Proof. It follows from

$$
\begin{aligned}
\left(M_{\left(g_{1}, g_{2}, g_{3}\right)}(X)\right)^{\lambda_{1}, \lambda_{2} \lambda_{3}} & =M_{\left(g_{1} \lambda_{1}, g_{2} \lambda_{2}, g_{3} \lambda_{3}\right)}(X) \\
\left(M_{\left(h_{1}, h_{2}, h_{3}\right)}(X)\right)^{\lambda_{1}, \lambda_{2} \lambda_{3}} & =M_{\left(h_{1} \lambda_{1}, h_{2} \lambda_{2}, h_{3} \lambda_{3}\right)}(X)
\end{aligned}
$$

and Lemma 2.1 that the proclaimed equality holds if and only if there exists $d \in S_{N}$ such that $d g_{i} \lambda_{i}=h_{i} \lambda_{i}$ holds for each $i=1,2,3$. This is equivalent to $h_{i}^{-1} d g_{i} \in S_{\lambda_{i}}$. Now the claim follows.

Lemma 2.4. In the above notations $P \in \mathcal{P}_{r}(N, N, N) \Rightarrow P^{\lambda_{1}, \lambda_{2}, \lambda_{3}} \in \mathcal{P}_{r}\left(n_{1}, n_{2}, n_{3}\right)$.

Proof. Given an $n_{1} \times n_{2} \times n_{3}$ tensor $A$ set

$$
\left(A^{\lambda_{1}, \lambda_{2}, \lambda_{3}}\right)_{i, j, k}=A_{i^{\lambda_{1}}, j^{\lambda_{2}}, k^{\lambda_{3}}}, \quad i, j, k \in[N] .
$$

Then $A^{\lambda_{1}, \lambda_{2}, \lambda_{3}}$ is an $N \times N \times N$ tensor, and it is easily seen that $\operatorname{rk}(A)=\operatorname{rk}\left(A^{\lambda_{1}, \lambda_{2}, \lambda_{3}}\right)$. It follows immediately from the definition of a $\left(\lambda_{1}, \lambda_{2}, \lambda_{3}\right)$-contraction that

$$
P^{\lambda_{1}, \lambda_{2}, \lambda_{3}}(A)=P\left(A^{\lambda_{1}, \lambda_{2}, \lambda_{3}}\right)
$$

and we are done.

For any multihomogeneous polynomial $P(X)$ the result of its polarization with a subsequent depolarization such that partitions $\mu=\left\{\lambda_{1}^{-1}(1), \ldots, \lambda_{1}^{-1}\left(n_{1}\right)\right\} ; \nu=$ $\left\{\lambda_{2}^{-1}(1), \ldots, \lambda_{2}^{-1}\left(n_{2}\right)\right\} ; \kappa=\left\{\lambda_{3}^{-1}(1), \ldots, \lambda_{3}^{-1}\left(n_{3}\right)\right\}$ coincides with $P(X)$ since the depolarization of each term from the sum (4) coincides with $M(X)$. Therefore, any multihomogeneous polynomial from $\mathcal{P}_{r}\left(n_{1}, n_{2}, n_{3}\right)$ can be obtained as the depolarization of a certain matching polynomial from $\mathcal{P}_{r}(N, N, N)$ where $N$ is the degree of the polynomial due to statement (2) of Lemma 2.2 . 


\section{A Reduction to the group algebra of $S_{n} \times S_{n}$}

Throughout the section we fix a number $d \in[n]$. Under a defect $d$ cubic determinant we mean any matching polynomial belonging the set $\mathcal{P}_{n-d}(n, n, n)$. This name is justified since such a polynomial vanishes at any tensor of the rank less or equal to $n-d$ (generalizing polynomials in matrices). A transition from $\mathcal{M}(n)$ to the group algebra $\mathbb{Q} S_{n}^{2}$ is provided by the linear space isomorphism

$$
F_{n}: \mathcal{M}(n) \rightarrow \mathbb{Q} S_{n}^{2}, \quad \sum_{g \in S_{n}^{2}} a(g) M_{g} \mapsto \sum_{g \in S_{n}^{2}} a(g) g .
$$

The vector space $\mathcal{M}(n)$ has a natural structure of a $\mathbb{Q} S_{n}^{3}$-module via the action of $S_{n}^{3}$ on matching monomials defined by $\left(M_{s}(X)\right)^{g}=M_{s g}(X)$. Writing a matching monomial in a reduced form $M_{s}, s \in S$ we obtain

$$
M_{\left(1, s_{2}, s_{3}\right)}^{\left(g_{1}, g_{2}, g_{3}\right)}(X)=M_{\left(g_{1}, s_{2} g_{2}, s_{3} g_{3}\right)}(X)=M_{\left(1, g_{1}^{-1} s_{2} g_{2}, g_{1}^{-1} s_{3} g_{3}\right)}(X)
$$

Thus the action of $S_{n}^{3}$ on matching monomials is equivalent to the action of $S_{n}^{3}$ on $S_{n} \times S_{n}$ definied by the formula

$$
\left(s_{2}, s_{3}\right)^{\left(g_{1}, g_{2}, g_{3}\right)}=\left(g_{1}^{-1} s_{2} g_{2}, g_{1}^{-1} s_{3} g_{3}\right) .
$$

Thus the permutation module arising from this action is isomorphic to $\mathcal{M}(n)$ and the subspace $V_{n, d}=F_{n}\left(\mathcal{P}_{n-d}(n, n, n)\right) \subseteq \mathbb{Q} S_{n}^{2}$ is a $\mathbb{Q} S_{n}^{3}$-submodule.

The action (7) is faithful and transitive. The normal subgroup $S$ of $S_{n}^{3}$ acts regularly on $S_{n}^{2}$ and can be identfied with the right regular action of $S_{n}^{2}$. The point stabilizer of $(1,1)$ coincides with the diagonal subgroup $D \leq S_{n}^{3}$. Thus $S_{n}^{3}=S \rtimes D$ and each element $g \in S_{n}^{3}$ has a unique decomposition into a product $g=\delta s$ with $\delta \in D$ and $s \in S$. Using this decompsition one can describe the above action (7) by the formula

$$
\iota\left(\chi^{g}\right)=\delta^{-1} \iota(\chi) \delta s, \text { where } \chi \in \mathbb{Q} S_{n}^{2} .
$$

The module corresponding to the action (7) is isomorphic (as a $\mathbb{Q} S_{n}^{3}$-module) to the right ideal generated by $\underline{D}$ (because $D$ is a point stabilizer of the action). A direct check shows that the linear map $\Phi$ defined by the formula

$$
\Phi\left(\underline{D}\left(g_{1}, g_{2}, g_{3}\right)\right)=\left(g_{1}^{-1} g_{2}, g_{1}^{-1} g_{3}\right)
$$

is an isomorphism between two $\mathbb{Q} S_{n}^{3}$-modules: $\underline{D} \cdot\left(\mathbb{Q} S_{n}^{3}\right)$ and $\mathbb{Q} S_{n}^{2} \cong \mathcal{M}(n)$. Since $G=S \rtimes D$, each element of the ideal $\underline{D} \cdot\left(\mathbb{Q} S_{n}^{3}\right)$ has a unique presentation as a product $\underline{D} \iota(\chi)$ with $\chi \in \mathbb{Q} S_{n}^{2}$. This yields the following formula

$$
\Phi(\underline{D} \iota(\chi) \cdot g)=\chi^{g} .
$$

For each $g \in S_{n}$ we define $\Delta_{1}(g)=(g, g), \Delta_{2}(g)=(g, 1), \Delta_{3}(g)=(1, g)$. Notice that each $\Delta_{i}: \mathbb{Q} S_{n} \rightarrow \mathbb{Q}\left(S_{n} \times S_{n}\right)$ is an algebra monomorphism. The importance of these monomorphisms follows from the following identity

$$
\Phi\left(x\left(g_{1}, g_{2}, g_{3}\right)\right)=\Delta_{1}\left(g_{1}^{-1}\right) \Phi(x) \Delta_{2}\left(g_{2}\right) \Delta_{3}\left(g_{3}\right), x \in \underline{D} \cdot \mathbb{Q} G .
$$

This identity implies that for any $x \in \underline{D} \cdot \mathbb{Q} G$ and arbitrary triple of elements $a, b, c \in \mathbb{Q} S_{n}$ it holds that

$$
\Phi(x \cdot(a \otimes b \otimes c))=\Delta_{1}\left(a^{-1}\right) \Phi(x) \Delta_{2}(b) \Delta_{3}(c) .
$$


Lemma 3.1. Let $\chi \in \mathbb{Q} S_{n}^{2}$ and $P(X)=F_{n}^{-1}(\chi)=\sum_{g \in S_{n}^{2}} \chi(g) M_{g}(X)$. Then $P$ is a cubic determinant of defect $d$ if and only if $\chi$ is a solution of the following system of linear equations:

$$
\underline{S}_{\lambda}^{2} \cdot \chi=0, \quad \lambda \in \Lambda(n, r)
$$

where $S_{\lambda}^{2}=S_{\lambda} \times S_{\lambda}$ and $r=n-d$.

Proof. To prove the sufficiency suppose that $\chi$ is a solution of system (10). Take an $n \times n \times n$ tensor $A$ of rank $\leq r$. Then there exist $n$-vectors $X^{(u)}, Y^{(u)}$ and $Z^{(u)}$, $u=1, \ldots, r$, such that

$$
A_{i j k}=\sum_{u=1}^{r} X_{i}^{(u)} Y_{j}^{(u)} Z_{k}^{(u)}, \quad i, j, k \in[n] .
$$

Without loss of generality we can assume that the elements of the vectors $X^{(u)}, Y^{(u)}$ and $Z^{(u)}$ are independent pairwise commuting variables. Then $P(A)$ is a polynomial on these variables, and for any monomial $M_{g}(X), g \in S_{n}^{2}$, of the polynomial $P(X)$ we have (see (3)):

$$
M_{g}(A)=\prod_{v=1}^{n}\left(\sum_{u=1}^{r} X_{v}^{(u)} Y_{v^{g_{1}}}^{(u)} Z_{v^{g_{2}}}^{(u)}\right) .
$$

This implies that any monomial of the polynomial $M_{g}(A)$, and hence any monomial of the polynomial $P(A)$, is uniquely determined by a map $\theta:[n] \rightarrow[r]$, in terms of which this monomial can be written as follows:

$$
M(g, \theta)=\prod_{v=1}^{n} X_{v}^{(\theta(v))} Y_{v^{g_{1}}}^{(\theta(v))} Z_{v^{g_{2}}}^{(\theta(v))} .
$$

Though not all of these monomials are distinct, we have

$$
P(A)=\sum_{g \in S_{n}^{2}} \chi(g) M_{g}(X)=\sum_{g \in S_{n}^{2}} \sum_{\theta:[n] \rightarrow[r]} \chi(g) M(g, \theta)
$$

where $\chi(g)$ is the coefficient of $\chi$ at $g$. For any two functions $\theta, \mu:[n] \rightarrow[r]$ the products $\prod_{v} X_{v}^{\theta(v)}, \prod_{v} X_{v}^{\mu(v)}$ are equal if and only if $\theta=\mu$. Therefore two monomials $M(g, \theta)$ and $M\left(g^{\prime}, \theta^{\prime}\right)$ are equal if and only if $\theta=\theta^{\prime}, g_{1}^{-1} \theta=g_{1}^{\prime-1} \theta^{\prime}, g_{2}^{-1} \theta=g_{2}^{\prime-1} \theta^{\prime}$. This shows that

$$
M(g, \theta)=M\left(g^{\prime}, \theta\right) \Longleftrightarrow g^{\prime} \in S_{\lambda}^{2} g
$$

where $\lambda$ is the partition of $[n]$ with $r^{\prime} \leq r$ non-empty classes $\theta^{-1}(i), i \in[r]$.

Thus given a right $S_{\lambda}^{2}$-coset $C$ in the group $S_{n}^{2}$, the monomial $M(g, \theta)$ does not depend on $g \in C$; we denote it by $M(C, \lambda)$. Then by (11) we obtain that

$$
P(A)=\sum_{\lambda \in \Lambda_{r}} \sum_{C \in C_{\lambda}} \sum_{g \in C} \chi(g) M(C, \lambda)=\sum_{\lambda \in \Lambda_{r}} \sum_{C \in C_{\lambda}}\left(\sum_{g \in C} \chi(g)\right) M(C, \lambda)
$$

where $C_{\lambda}$ is the set of all $S_{\lambda}^{2}$-cosets in $S_{n}^{2}$, and $\Lambda_{r}$ is the set of all partitions of $[n]$ into at most $r$ classes. Thus $P$ is of defect $d$ if and only if for any $\chi$ the following condition holds:

$$
\forall \lambda \in \Lambda_{r} \quad \forall C \in C_{\lambda} \sum_{g \in C} \chi(g)=0
$$


In its turn, (12) is equivalent to the following one

$$
\forall \lambda \in \Lambda_{r} \quad \underline{S}_{\lambda}^{2} \cdot \chi=0 .
$$

Therefore to finish the proof it remains to show that

$$
\forall \lambda \in \Lambda_{r} \quad \underline{S}_{\lambda}^{2} \cdot \chi=0 \Longleftrightarrow \forall \lambda \in \Lambda(n, r) \quad \underline{S}_{\lambda}^{2} \cdot \chi=0 .
$$

One direction follows immediately from the inclusion $\Lambda(n, r) \subseteq \Lambda_{r}$. To prove the other direction pick an arbitrary $\lambda \in \Lambda_{r}$. There exists a refinement $\mu \in \Lambda(n, r)$ of $\lambda$. Then $S_{\mu}^{2} \leq S_{\lambda}^{2}$, implying $\underline{S}_{\lambda}^{2} \cdot \underline{S}_{\mu}^{2}=k \underline{S}_{\lambda}^{2}$ with $k=\left|S_{\mu}\right|^{2}$. Now we obtain

$$
\underline{S}_{\mu}^{2} \cdot \chi=0 \Rightarrow \underline{S}_{\lambda}^{2} \cdot \underline{S}_{\mu}^{2} \cdot \chi=0 \Rightarrow k \underline{S}_{\lambda}^{2} \cdot \chi=0 \Rightarrow \underline{S}_{\lambda}^{2} \cdot \chi=0 .
$$

Lemma 3.1 shows that $V_{n, d}$ coincides with the solutions of the linear system defined by (10). Clearly that $V_{n, d}$ set is a right ideal of the group algebra $\mathbb{Q} S_{n}^{2}$. Lemma 3.2 below enables us to reduce the system (10) to a single linear equation by means of the element

$$
\zeta=\zeta_{n, d}=\sum_{\lambda \in \Lambda(n, n-d)} \underline{S}_{\lambda}^{2} .
$$

For every partititon $\lambda \in \Lambda(n)$ and a permutation $g \in S_{n}$, it holds that $g^{-1} S_{\lambda} g=$ $S_{g^{-1} \lambda}$. This implies

$$
(g, g)^{-1} \zeta(g, g)=\sum_{\lambda \in \Lambda(n, n-d)} \underline{S}_{g^{-1} \lambda}^{2}=\zeta .
$$

Therefore the coefficients of $\zeta$ are constant on the orbits of the coordinatewise conjugacy action of $S_{n}$ on $S_{n}^{2}$.

To compute the element $\zeta_{n, 1}$, we note that every partition $\lambda \in \Lambda(n, n-1)$ has exactly one class of size 2 and $n-2$ singleton classes. Therefore $S_{\lambda}^{2}$ is a Klein group whose non-identity elements are $(t, t),\left(1_{n}, t\right)$ and $\left(t, 1_{n}\right)$ for some transposition $t \in S_{n}$ where $1_{n}$ is the identity permutation in $S_{n}$. It follows that

$$
\zeta_{n, 1}=\left(\begin{array}{l}
n \\
2
\end{array}\right) \underline{1}_{n}+\underline{C}_{1}+\underline{C}_{2}+\underline{C}_{3}
$$

where $C_{1}=\operatorname{Diag}(T \times T), C_{2}=\left\{1_{n}\right\} \times T$ and $C_{3}=T \times\left\{1_{n}\right\}$ with $T=T_{n}$ being the set of transpositions in $S_{n}$.

Lemma 3.2. In the above notations $V_{n, d}=\left\{\chi \in \mathbb{Q} S_{n}^{2}: \zeta \cdot \chi=0\right\}$.

Proof. Clearly, $\zeta \cdot \chi=0$ for all $\chi \in V_{n, d}$. Conversely, let $\chi \in \mathbb{Q} S_{n}^{2}$ be such that $\zeta \cdot \chi=0$. We have to verify that $\underline{S}_{\lambda}^{2} \cdot \chi=0$ for all $\lambda \in \Lambda(n, r)$ where $r=n-d$. However,

$$
\zeta \cdot \chi=0 \Rightarrow\langle\zeta \cdot \chi, \chi\rangle=0 \Rightarrow \sum_{\lambda \in \Lambda(n, r)}\left\langle\underline{S}_{\lambda}^{2} \cdot \chi, \chi\right\rangle=0
$$

where $\langle\cdot, \cdot\rangle$ is the standard scalar product in $\mathbb{Q} S_{n}^{2}$. Since $S_{\lambda}^{2}$ is a subgroup of $S_{n}^{2}$, the quadratic form $\left\langle\underline{S}_{\lambda}^{2} \cdot \chi, \chi\right\rangle$ is positive semidefinite. Hence the right-hand side equation in (16) implies that $\left\langle\underline{S}_{\lambda}^{2} \cdot \chi, \chi\right\rangle=0$, and hence $\underline{S}_{\lambda}^{2} \cdot \chi=0$ for all $\lambda \in \Lambda(n, r)$. 
We complete the section by giving a necessary and sufficient condition for a matching polynomial to have a nonzero contraction in terms of the Young subgroups of the corresponding partitions. For an element $\chi \in \mathbb{Q} S_{n}^{2}$ set

$$
\chi^{\lambda_{1}, \lambda_{2}, \lambda_{3}}=\Delta_{1}\left(\underline{S}_{\lambda_{1}}\right) \cdot \chi \cdot \Delta_{2}\left(\underline{S}_{\lambda_{2}}\right) \cdot \Delta_{3}\left(\underline{S}_{\lambda_{3}}\right) .
$$

This element will be called the $\left(\lambda_{1}, \lambda_{2}, \lambda_{3}\right)$-contraction of $\chi$.

Lemma 3.3. Let $P \in \mathcal{M}(n), \chi=F_{n}(P)$ and $\lambda_{1}, \lambda_{2}, \lambda_{3} \in \Lambda_{n}$. Then for $S_{\lambda}=$ $S_{\lambda_{1}} \times S_{\lambda_{2}} \times S_{\lambda_{2}}$ we have

$$
P^{\lambda_{1}, \lambda_{2}, \lambda_{3}} \neq 0 \quad \Longleftrightarrow \quad \underline{D} \cdot \iota(\chi) \cdot \underline{S_{\lambda}} \neq 0 \Longleftrightarrow \quad \chi^{\lambda_{1}, \lambda_{2}, \lambda_{3}} \neq 0 .
$$

Proof. It is enough to prove the first equivalence, since the second one follows from (9):

$\Phi\left(\underline{D} \cdot \iota(\chi) \cdot \underline{S_{\lambda}}\right)=\Delta_{1}\left(\underline{S}_{\lambda_{1}}\right) \cdot \Phi(\underline{D} \cdot \iota(\chi)) \cdot \Delta_{2}\left(\underline{S}_{\lambda_{2}}\right) \cdot \Delta_{3}\left(\underline{S}_{\lambda_{3}}\right)=\Delta_{1}\left(\underline{S}_{\lambda_{1}}\right) \cdot \chi \cdot \Delta_{2}\left(\underline{S}_{\lambda_{2}}\right) \cdot \Delta_{3}\left(\underline{S}_{\lambda_{3}}\right)$.

By the definition of the mapping $F_{n}$ we have

$$
\begin{gathered}
P(X)=\sum_{g \in S_{n}^{2}} \chi(g) M_{g}(X)=\sum_{g \in S_{n}^{2}} \chi(g) M_{\iota(g)}(X) \Rightarrow \\
P(X)^{\left(\lambda_{1}, \lambda_{2}, \lambda_{3}\right)}=\sum_{g \in S} \chi\left(\iota^{-1}(g)\right) M_{g}(X)^{\left(\lambda_{1}, \lambda_{2}, \lambda_{3}\right)} .
\end{gathered}
$$

By Lemma $2.1 M_{g}(X)^{\left(\lambda_{1}, \lambda_{2}, \lambda_{3}\right)}=M_{h}(X)^{\left(\lambda_{1}, \lambda_{2}, \lambda_{3}\right)}$ iff $D g S_{\lambda}=D h S_{\lambda}$. Thus the monomial $M_{h}(X)^{\left(\lambda_{1}, \lambda_{2}, \lambda_{3}\right)}$ does not depend on a choice of $h$ in the double coset $C=D g S_{\lambda}$. Denoting this monomial by $M_{C}(Y)$ we can write ${ }^{1}$

$$
P(X)^{\left(\lambda_{1}, \lambda_{2}, \lambda_{3}\right)}=\sum_{C \in D \backslash S / S_{\lambda}}\left(\sum_{g \in C \cap S} \chi\left(\iota^{-1}(g)\right)\right) M_{C}(Y) .
$$

Let $c_{g}, g \in S_{n}^{3}$ denote the number of pairs $(d, h) \in D \times S_{\lambda}$ satisfying $d g h=g$. Notice that $c_{g}=c_{g^{\prime}}$ for any $g^{\prime} \in D g S_{\lambda}$, and, $c_{g}=\frac{|D|\left|S_{\lambda}\right|}{\left|D g S_{\lambda}\right|}$. Thus $\underline{D} \cdot g \cdot \underline{S_{\lambda}}=\frac{|D|\left|S_{\lambda}\right|}{\left|D g S_{\lambda}\right|} \underline{D g S_{\lambda}}$ implying

$\underline{D} \cdot \iota(\chi) \cdot \underline{S_{\lambda}}=\underline{D} \cdot\left(\sum_{g \in S} \chi\left(\iota^{-1}(g)\right) g\right) \cdot \underline{S_{\lambda}}=\sum_{C \in D \backslash S / S_{\lambda}} \frac{|D|\left|S_{\lambda}\right|}{|C|}\left(\sum_{g \in C \cap S} \chi\left(\iota^{-1}(g)\right)\right) \underline{C}$.

Now it follows from $\frac{|D|\left|S_{\lambda}\right|}{|C|}>0$ that both expressions $P(X)^{\left(\lambda_{1}, \lambda_{2}, \lambda_{3}\right)}$ and $\underline{D} \cdot \iota(\chi) \cdot \underline{S_{\lambda}}$ are nonzero simultenously.

\section{A decomposition of the Cubic Determinant space via a Schur Ring}

Throughout this section we fix positive integers $n$ and $d \in[n]$, and set $V=V_{n, d}$.

The ideal $\underline{D} \cdot \mathbb{Q} G$ is generated by an idempotent $e_{D}=\frac{1}{|D|} \underline{D}$. Therefore, its ring of endomorphisms coincides with $e_{D}(\mathbb{Q} G) e_{D}$. In order to describe the image $\Phi\left(e_{D}(\mathbb{Q} G) e_{D}\right)$ inside $\mathbb{Q} S_{n}^{2}$ we introduce the vector space $\mathcal{A}$ spanned by the simple quantities $\underline{s}^{D}, s=\left(s_{2}, s_{3}\right) \in S_{n}^{2}$. Recall that $s^{D}=\left\{s^{d} \mid d \in D\right\}$ and by (8) $\left(s_{2}, s_{3}\right)^{(g, g, g)}=\left(g^{-1} s_{2} g, g^{-1} s_{3} g\right)$. Notice that the diagonal subgroup $D$ acts on $S_{n}^{2}$ as an automorphism group and $\mathcal{A}=\left\{\chi \in \mathbb{Q} S_{n}^{2} \mid \forall_{d \in D} \chi^{d}=\chi\right\}$. In other words, $\mathcal{A}$

\footnotetext{
${ }^{1}$ Below $D \backslash S / S_{\lambda}$ stands for the set of double cosets $D s S_{\lambda}, s \in S$.
} 
is a $D$-fixed point subalgebra of $\mathbb{Q} S_{n}^{2}$. This algebra is also a Schur ring (see [26, Chapter IV]) which arises from the action of $\mathbb{Q} S_{n}^{3}$ on $S_{n}^{2}$.

Lemma 4.1. $\mathcal{A}=\Phi\left(e_{D}\left(\mathbb{Q} S_{n}^{2}\right) e_{D}\right)$ and $\widetilde{\Phi}=|D| \Phi$ is an algebra isomorphism between $e_{D}\left(\mathbb{Q} S_{n}^{2}\right) e_{D}$ and $\mathcal{A}$.

Proof. We can replace $e_{D}\left(\mathbb{Q} S_{n}^{2}\right) e_{D}$ by $\underline{D}(\mathbb{Q} G) \underline{D}$, because those subspaces are equal.

Pick an arbitrary $s \in S_{n}^{2}$. Then

$$
\Phi(\underline{D} \iota(s) \underline{D})=\sum_{d \in D} \Phi(\underline{D} \iota(s) d)=\sum_{d \in D} s^{d} \in \mathcal{A}
$$

implying $\mathcal{A}=\Phi(\underline{D}(\mathbb{Q} G) \underline{D})$ (recall that the elements $\left.\underline{D} \iota(s)(D), s \in S_{n}^{2} \operatorname{span} \underline{D}(\mathbb{Q} G) \underline{D}\right)$. To prove that $\widetilde{\Phi}$ is an isomorphism it is sufficient to show that

$$
\widetilde{\Phi}(x y)=\widetilde{\Phi}(x) \widetilde{\Phi}(y)
$$

holds for any pair $x, y$ of the form $x=\underline{D} \iota(s) \underline{D}, y=\underline{D} \iota(t) \underline{D}$ where $s, t \in S_{n}^{2}$. First we notice that for any $r \in S_{n}^{2}$ it holds that

$$
\underline{D} \iota(r) \underline{D}=\underline{D}\left(\sum_{d \in D} d^{-1} \iota(r) d\right)=\underline{D}\left(\sum_{d \in D} \iota\left(r^{d}\right)\right)=\left|C_{D}(r)\right| \cdot \underline{D} \cdot \iota\left(\underline{r^{D}}\right),
$$

where $C_{D}(r)=\left\{d \in D \mid r^{d}=r\right\}$. Thus $\widetilde{\Phi}(x)=\left|D \| C_{D}(s)\right| \underline{s^{D}}$ and $\widetilde{\Phi}(y)=$ $|D|\left|C_{D}(t)\right| \underline{t^{D}}$. Now we have

$$
\begin{gathered}
\widetilde{\Phi}(x y)=|D|((\underline{D} \iota(s) \underline{D})(\underline{D} \iota(t) \underline{D}))=|D|^{2} \Phi(\underline{D} \iota(s) \underline{D} \iota(t) \underline{D})= \\
|D|^{2}\left|C_{D}(t)\right| \Phi\left(\underline{D} \iota(s) \underline{D} \iota\left(\underline{t^{D}}\right)\right)=|D|^{2}\left|C_{D}(t)\right|\left|C_{D}(s)\right| \Phi\left(\underline{D} \iota\left(\underline{s^{D}}\right) \iota\left(\underline{t^{D}}\right)\right)= \\
=|D|^{2}\left|C_{D}(t)\right|\left|C_{D}(s)\right| \Phi\left(\underline{D} \iota\left(\underline{s^{D}} \cdot \underline{t^{D}}\right)\right)=|D|^{2}\left|C_{D}(t)\right|\left|C_{D}(s)\right| \underline{s^{D}} \cdot \underline{t^{D}}=\widetilde{\Phi}(x) \widetilde{\Phi}(y)
\end{gathered}
$$

The statement below gives a characterization of $\mathbb{Q} S_{n}^{3}$-submodules of $\mathbb{Q} S_{n}^{2}$.

Lemma 4.2. A vector subspace $W \subseteq \mathbb{Q} S_{n}^{2}$ is a $\mathbb{Q} S_{n}^{3}$-submodule iff it is a $D$ invariant right ideal of the group algebra $\mathbb{Q} S_{n}^{2}$.

Proof. follows immediately from (7).

Since $V$ is a $\mathbb{Q} S_{n}^{3}$ submodule of $\mathbb{Q} S_{n}^{2}$, it is a $D$-invariant right ideal of the group algebra $\mathbb{Q} S_{n}^{2}$.

Since $\mathbb{Q} S_{n}^{2}$ is a semisimple module of $\mathbb{Q} S_{n}^{3}$, the submodule $V$ is a direct sum of irreducible ones. In order to study irreducible components of $\mathbb{Q} S_{n}^{2}$ we will use the algebra $\mathcal{A}$. Denote by $\operatorname{Ir}(\mathcal{A})$ the set of all $\mathbb{Q}$-irreducible characters of $\mathcal{A}$. As we will see below (Lemma 4.4) these characters are absolutely irreducible. By the Wedderburn theorem this implies that

$$
\mathcal{A}=\bigoplus_{\pi \in \operatorname{lrr}(\mathcal{A})} \mathcal{A}_{\pi}
$$

where $\mathcal{A}_{\pi}$ is a simple algebra isomorphic to $\operatorname{Mat}_{n_{\pi}}(\mathbb{Q})$ with $n_{\pi}=\pi\left(1_{S_{n}^{2}}\right)$. The following statement shows how the space of cubic determinants of defect $d$ can be constructed from spaces $V \cap \mathcal{A}_{\pi}$ where $V=V_{n, d}$. 
Lemma 4.3. Let $W \subseteq \mathbb{Q} S_{n}^{2}$ be a $\mathbb{Q} S_{n}^{3}$-submodule. Then the linear space $W$ is generated (as a right ideal of the algebra $\mathbb{Q} S_{n}^{2}$ ) by the linear space $W \cap \mathcal{A}$. Moreover,

$$
W \cap \mathcal{A}=\bigoplus_{\pi \in \operatorname{Irr}(\mathcal{A})} W \cap \mathcal{A}_{\pi}
$$

Proof. Since $\mathbb{Q} S_{n}^{3}$ is a semisimple algebra, every $\mathbb{Q} S_{n}^{3}$-module is a direct sum of the irreducible ones. Thus it is enough to prove the statement for irreducible submodules. Let now assume that $W$ is irreducible (minimal) submodule of $\mathbb{Q} S_{n}^{2}$. Applying module isomorphism $\Phi^{-1}$ we obtain that $J=\Phi^{-1}(W)$ is a minimal right ideal of $R=\mathbb{Q} S_{n}^{3}$ contained in $e_{D} R$. It follows from semisimplicity of $R$ that $J=e R$ for some idempotent $e \in R$. The inclusion $e_{D} R \supseteq e R$ implies $e_{D} e=e$. An element $e e_{D}$ is non-zero, because $e e_{D} e=e \neq 0$. Now, by minimality of $J=e R$, we obtain $e e_{D} R=e R$. Together with $e e_{D} \in\left(e_{D} R e_{D} \cap J\right)$ this implies $\left(e_{D} R e_{D} \cap J\right) R=J$. In other words, the set $e_{D} R e_{D} \cap J$ generate $J$ as ideal. Applying now $\Phi$ to both sides, we conclude that $\Phi\left(e_{D} R e_{D}\right) \cap W=\mathcal{A} \cap W$ generate $W$ as a $\mathbb{Q} S_{n}^{3}$-module, that is $W=\operatorname{Span}\left\{(\mathcal{A} \cap W)^{g} \mid g \in S_{n}^{3}\right\}$. Now it follows from $(\mathcal{A} \cap W)^{D}=\mathcal{A} \cap W$ that $W=(\mathcal{A} \cap W)\left(\mathbb{Q} S_{n}^{2}\right)$. To prove the second statement we note that $W \cap \mathcal{A}$ is a right $\mathcal{A}$-ideal. Therefore (18) immediately follows from (17).

Given a character $\pi \in \operatorname{Irr}(\mathcal{A})$ denote by $e_{\pi}$ the central primitive idempotent ${ }^{2}$ of the algebra $\mathcal{A}$ that corresponds to $\pi$. Then $\mathcal{A}_{\pi}=e_{\pi} \cdot \mathcal{A}$. Therefore to study the linear space $V \cap \mathcal{A}_{\pi}$ we find the explicit expression for the idempotent $e_{\pi}$ in terms of the irreducible characters of the group $S_{n}$. For this purpose let us fix some notations. First, given $\rho \in \operatorname{Irr}\left(S_{n}\right)$ we denote by $e_{\rho}$ the central primitive idempotent of the algebra $\mathbb{Q} S_{n}$ that corresponds to $\rho .^{3}$ The rationality of $\rho$ implies that

$$
e_{\rho}=\frac{\rho\left(1_{n}\right)}{n !} \cdot \sum_{g \in S_{n}} \rho(g) g
$$

Second, given any two irreducible characters $\mu, \nu \in \operatorname{Irr}\left(S_{n}\right)$ we have $\mu \cdot \nu=\sum_{\rho} k_{\mu, \nu}^{\rho} \rho$ where $\rho$ runs over the set $\operatorname{Irr}\left(S_{n}\right)$ and $k_{\mu, \nu}^{\rho}$ is a nonnegative integer called the Kronecker coefficient [3].

Lemma 4.4. Given a character $\pi \in \operatorname{Irr}(\mathcal{A})$ there exist uniquely determined characters $\rho, \mu, \nu \in \operatorname{Irr}\left(S_{n}\right)$ such that $k_{\mu, \nu}^{\rho} \neq 0$ and $e_{\pi}=e_{\rho, \mu, \nu}$ where

$$
e_{\rho, \mu, \nu}=\Delta_{1}\left(e_{\rho}\right) \cdot \Delta_{2}\left(e_{\mu}\right) \cdot \Delta_{3}\left(e_{\nu}\right) .
$$

Proof. By Lemma $4.1 e_{\pi}=\widetilde{\Phi}(f)$ for some central idempotent $f \in e_{D}(\mathbb{Q} G) e_{D}$. Pick a primitive central idempotent $g$ of the algebra $\left(1-e_{D}\right)\left(\mathbb{Q} S_{n}^{2}\right)\left(1-e_{D}\right)$. Then $f+g$ is a primitive central idempotent of $\mathbb{Q} S_{n}^{3}$ and $f=(f+g) e_{D}$. Since each primitive central idempotent of $\mathbb{Q} S_{n}^{3}$ has a form $e_{\pi^{\prime}}$ for some $\pi^{\prime} \in \operatorname{Irr}\left(S_{n}^{3}\right)$, we conclude that $f=e_{D} e_{\pi^{\prime}}$.

Notice that $e_{D} e_{\pi^{\prime}} \neq 0$ if and only if $\sum_{g \in D} \pi^{\prime}(g) \neq 0$. Each irreducible character $\pi^{\prime}$ of $\mathbb{Q} S_{n}^{3}$ is a tensor product of irreducible characters $\rho, \mu, \nu$ of $S_{n}$, that is

\footnotetext{
${ }^{2}$ Recall that a central idempotent is called primitive if it cannot be decomposed into anorthogonal sum central idempotents.

${ }^{3}$ Here and below we are working in the algebra $\mathbb{Q} S_{n}$, because the irreducible representations of $S_{n}$ over $\mathbb{Q}$ are absolutely irreducible.
} 
$\pi^{\prime}\left(\left(g_{1}, g_{2}, g_{3}\right)\right)=\rho\left(g_{1}\right) \mu\left(g_{2}\right) \nu\left(g_{3}\right)$ and $e_{\pi^{\prime}}=e_{\rho} \otimes e_{\mu} \otimes e_{\nu}$. Therefore $e_{D} e_{\pi^{\prime}} \neq 0$ if and only if

$$
0 \neq \sum_{d \in S_{n}} \pi^{\prime}((d, d, d))=\sum_{d \in S_{n}} \rho(d) \mu(d) \nu(d)=n ! \kappa_{\mu, \nu}^{\rho} .
$$

Thus $e_{\pi}=\widetilde{\Phi}\left(e_{D}\left(e_{\rho} \otimes e_{\mu} \otimes e_{\nu}\right)\right)=\Delta_{1}\left(e_{\rho}\right) \Delta_{2}\left(e_{\mu}\right) \Delta_{3}\left(e_{\nu}\right)$.

Let us consider the contraction of a cubic determinants (see Section 2 and Lemma 3.3). For this purpose denote by $\boldsymbol{\lambda}_{\rho}$ the Young diagram corresponding a character $\rho \in \operatorname{Irr}\left(S_{n}\right)$, and for a partition $\lambda \in \Lambda(n)$ by $\pi_{\lambda}$ the permutation character of the group $S_{n}$ acting on the right cosets of $S_{\lambda}$ by multiplications. Then by [11, Corollary 2.2.22] we have

$$
\left\langle\pi_{\lambda}, \rho\right\rangle \neq 0 \quad \Leftrightarrow \quad \lambda_{\rho} \unrhd[\lambda]
$$

where $\unrhd$ denotes the partial order on $\boldsymbol{\Lambda}(n)$ in which $\boldsymbol{\lambda} \unrhd \boldsymbol{\mu}(\boldsymbol{\lambda}$ dominates $\boldsymbol{\mu})$ if and only if for all $i$ the inequality $\sum_{j=1}^{i} \boldsymbol{\lambda}_{j} \geq \sum_{j=1}^{i} \boldsymbol{\mu}_{j}$ holds.

Lemma 4.5. Given $\rho, \lambda, \mu \in \operatorname{Irr}\left(S_{n}\right)$ and $\boldsymbol{\lambda}_{1}, \boldsymbol{\lambda}_{2}, \boldsymbol{\lambda}_{3} \in \boldsymbol{\Lambda}(n)$ set $e=e_{\rho, \mu, \nu}$ and set $\Lambda_{i}=\left\{\lambda_{i} \in \Lambda(n):\left[\lambda_{i}\right]=\boldsymbol{\lambda}_{i}\right\}, i=1,2,3$. Then

(1) if $\boldsymbol{\lambda}_{1} \unrhd \boldsymbol{\lambda}_{\rho}$ or $\boldsymbol{\lambda}_{2} \unrhd \boldsymbol{\lambda}_{\mu}$ or $\boldsymbol{\lambda}_{3} \unrhd \boldsymbol{\lambda}_{\nu}$, then $\chi^{\lambda_{1}, \lambda_{2}, \lambda_{3}}=0$ for all $\chi \in e \mathbb{Q} S_{n}^{2}$ and all $\lambda_{i} \in \Lambda_{i}$

(2) if $\boldsymbol{\lambda}_{1} \unrhd \boldsymbol{\lambda}_{\rho}$ and $\boldsymbol{\lambda}_{2} \unrhd \boldsymbol{\lambda}_{\mu}$ and $\boldsymbol{\lambda}_{3} \unrhd \boldsymbol{\lambda}_{\nu}$ and $k_{\mu, \nu}^{\rho} \neq 0$, then $e^{\lambda_{1}, \lambda_{2}, \lambda_{3}} \neq 0$ for some $\lambda_{i} \in \Lambda_{i}$.

Proof. To prove statement (1) suppose that $\boldsymbol{\lambda}_{1} \unrhd \boldsymbol{\lambda}_{\rho}$ or $\boldsymbol{\lambda}_{2} \unrhd \boldsymbol{\lambda}_{\mu}$ or $\boldsymbol{\lambda}_{3} \unrhd \boldsymbol{\lambda}_{\nu}$. Then by (21) for any partitions $\lambda_{1} \in \Lambda_{1}, \lambda_{2} \in \Lambda_{2}$ and $\lambda_{3} \in \Lambda_{3}$ we have

$$
\left\langle\pi_{\lambda_{1}}, \rho\right\rangle=0 \quad \text { or } \quad\left\langle\pi_{\lambda_{2}}, \mu\right\rangle=0 \quad \text { or } \quad\left\langle\pi_{\lambda_{3}}, \nu\right\rangle=0 .
$$

On the other hand, the idempotents of the algebra $\mathbb{Q} S_{n}$ corresponding the permutation characters $\pi_{\lambda_{1}}, \pi_{\lambda_{2}}$ and $\pi_{\lambda_{3}}$ coincide up to multiple $1 / a_{\lambda}$ where $a_{\lambda}=\left|S_{\lambda_{1}}\right|$, with the elements $\underline{S}_{\lambda_{1}}, \underline{S}_{\lambda_{2}}$ and $\underline{S}_{\lambda_{3}}$ respectively. Thus one of the elements $\underline{S}_{\lambda_{1}} \cdot e_{\rho}$, $\underline{S}_{\lambda_{2}} \cdot e_{\mu}, \underline{S}_{\lambda_{3}} \cdot e_{\nu}$ is equal to 0 . Therefore due to (20) for any $g \in S_{n}^{2}$ we obtain that

$$
(e \cdot g)^{\lambda_{1}, \lambda_{2}, \lambda_{3}}=\Delta_{1}\left(\underline{S_{\lambda_{1}}} e_{\rho}\right) \cdot \Delta_{2}\left(\underline{\left\{g_{1}\right\}} \cdot \underline{S_{\lambda_{2}}} e_{\mu}\right) \cdot \Delta_{3}\left(\underline{\left\{g_{2}\right\}} \cdot \underline{S_{\lambda_{3}}} e_{\nu}\right)=0
$$

(here we used the facts that the elements $\Delta_{2}(X)$ and $\Delta_{3}(Y)$ commute each to other for all $X, Y \in \mathbb{Q} S_{n}$, and that the elements $e_{\rho}, e_{\mu}$ and $e_{\nu}$ belong to the center of the algebra $\mathbb{Q} S_{n}$ ). Now, statement (1) follows from Lemma 3.3.

To prove statement (2) suppose that $\boldsymbol{\lambda}_{1} \unrhd \boldsymbol{\lambda}_{\rho}$ and $\boldsymbol{\lambda}_{2} \unrhd \boldsymbol{\lambda}_{\mu}$ and $\boldsymbol{\lambda}_{3} \unrhd \boldsymbol{\lambda}_{\nu}$. Then again as above from (21) it follows that

$$
\sum_{\lambda_{1}} \underline{S_{\lambda_{1}}} \cdot e_{\rho}=a_{\lambda} \cdot e_{\rho}, \quad \sum_{\lambda_{2}} \underline{S_{\lambda_{2}}} \cdot e_{\mu}=a_{\lambda} \cdot e_{\mu}, \quad \sum_{\lambda_{3}} \underline{S_{\lambda_{3}}} \cdot e_{\nu}=a_{\lambda} \cdot e_{\rho},
$$

where $\lambda_{i}$ runs over all the partitions in $\Lambda_{i}$. Since $k_{\rho, \mu}^{\nu} \neq 0$ by Lemma 4.4 we have

$$
0 \neq a_{\lambda}^{3} e^{\lambda_{1}, \lambda_{2}, \lambda_{3}}=\sum_{\lambda_{1}} \Delta_{1}\left(\underline{S_{\lambda_{1}}} \cdot e_{\rho}\right) \cdot \sum_{\lambda_{2}} \Delta_{2}\left(\underline{S_{\lambda_{2}}} \cdot e_{\mu}\right) \cdot \sum_{\lambda_{3}} \Delta_{3}\left(\underline{S_{\lambda_{3}}} \cdot e_{\nu}\right) .
$$

Since any element of the form $\Delta_{1}\left(\underline{S_{\lambda_{1}}} \cdot e_{\rho}\right) \cdot \Delta_{2}\left(\underline{S_{\lambda_{2}}} \cdot e_{\mu}\right) \cdot \Delta_{3}\left(\underline{S_{\lambda_{3}}} \cdot e_{\nu}\right)$ is an idempotent in $\mathbb{Q} S_{n}^{2}$, at least one of them is not zero, and we are done.

It is easily seen that any $\left(\lambda_{1}, \lambda_{2}, \lambda_{3}\right)$-contraction of the element $e_{\rho, \mu, \nu}$ is a nonnegative multiple of an idempotent of the algebra $\mathbb{Q} S_{n}^{2}$. Therefore under the condition of statement (2) of Lemma 4.5 the sum of all $\left(\lambda_{1}, \lambda_{2}, \lambda_{3}\right)$-contractions of $e_{\rho, \mu, \nu}$ is 
not zero. Thus, summing up the results from Lemmas 4.3, 4.4 and 4.5 we come to the following statement.

Theorem 4.6. The linear space $V_{n, d}$ is generated as a right ideal of the algebra $\mathbb{Q} S_{n}^{2}$ by the sets

$$
V_{\rho, \mu, \nu}=\left\{\chi \in \mathcal{A}_{\rho, \mu, \nu}: \zeta_{n, d} \cdot e_{\rho, \mu, \nu} \cdot \chi=0\right\}
$$

where $\rho, \mu, \nu$ run over the set $\operatorname{Irr}\left(S_{n}\right)$ with $k_{\mu, \nu}^{\rho} \neq 0$. In particular, $V_{\rho, \mu, \nu}=\mathcal{A}_{\rho, \mu, \nu}$ if and only if $e_{\rho, \mu, \nu} \in V_{n, d}$. In the latter case the sum of all $\left(\lambda_{1}, \lambda_{2}, \lambda_{3}\right)$-contractions of the element $e_{\rho, \mu, \nu}$ is not zero whenever the partitions $\lambda_{1}, \lambda_{2}$ and $\lambda_{3}$ have the same Young diagram and this diagram dominates the diagrams $\boldsymbol{\lambda}_{\rho}, \boldsymbol{\lambda}_{\mu}$ and $\boldsymbol{\lambda}_{\mu}$.

Given a Young diagram $\boldsymbol{\lambda}$ denote by $r(\boldsymbol{\lambda})$ the number of rows of $\boldsymbol{\lambda}$. Then it is easily seen that $\boldsymbol{\lambda} \unrhd \boldsymbol{\mu}$ only if $r(\boldsymbol{\lambda}) \leq r(\boldsymbol{\mu})$. Therefore the following statement immediately follows from statement (1) of Lemma 4.5.

Corollary 4.7. Let $\rho, \mu, \nu \in \operatorname{Irr}\left(S_{n}\right)$ and $\lambda_{1}, \lambda_{2}, \lambda_{3} \in \Lambda(n)$. Then any cubic determinant in $\mathcal{M}(n)$ that corresponds to an element of $V_{\rho, \mu, \nu}$ has a nonzero $\left(\lambda_{1}, \lambda_{2}, \lambda_{3}\right)$ contraction only if $r\left(\lambda_{1}\right) \leq r\left(\boldsymbol{\lambda}_{\rho}\right), r\left(\lambda_{2}\right) \leq r\left(\boldsymbol{\lambda}_{\mu}\right)$ and $r\left(\lambda_{3}\right) \leq r\left(\boldsymbol{\lambda}_{\nu}\right)$.

\section{Cubic Determinants of Defect 1}

In this section we refine Theorem 4.6 for the case $d=1$. To compute the elements $\zeta \cdot e_{\pi}$ with $\zeta=\zeta_{n, 1}$ and $\pi \in \operatorname{Irr}(\mathcal{A})$ we need the following auxiliary lemma.

Lemma 5.1. Let $C$ and $\rho$ be a conjugacy class and an irreducible character of $S_{n}$, respectively. Then

(1) $\underline{C} \cdot e_{\rho}=\frac{|C| \rho(g)}{\rho\left(1_{n}\right)} e_{\rho}$ for any $g \in C$,

(2) $\Delta_{i}(\underline{C}) \cdot \Delta_{j}\left(e_{\rho}\right)=\Delta_{j}\left(e_{\rho}\right) \cdot \Delta_{i}(\underline{C})$ for $i, j=1,2,3$.

Proof. To prove statement $(1)^{4}$ we note that the element $\underline{C}$ belongs to the center of the group algebra $\mathbb{Q} S_{n}$. Therefore $\chi:=\underline{C} \cdot e_{\rho}=a \cdot e_{\rho}$ for some $a \in \mathbb{Q}$. After comparing the coefficients in both sides of the latter equality at $1_{n}$, we get that $a=\chi\left(1_{n}\right) / e_{\rho}\left(1_{n}\right)$. However, by (19) we have

$$
\chi\left(1_{n}\right)=|C| \rho(g) \frac{\rho\left(1_{n}\right)}{n !} \quad \text { and } \quad e_{\rho}\left(1_{n}\right)=\frac{\left(\rho\left(1_{n}\right)\right)^{2}}{n !}
$$

where $g \in C$. Thus $a=|C| \rho(g) / \rho\left(1_{n}\right)$ and we are done.

Statement (2) is obvious whenever $\{i, j\} \subset\{2,3\}$ or $i=j=1$. Suppose that $i=1$ and $j=2$ (the remaining three cases are proved in a similar way). Then

$$
\begin{gathered}
\frac{n !}{\rho\left(1_{n}\right)} \Delta_{1}(\underline{C}) \cdot \Delta_{2}\left(e_{\rho}\right)=\sum_{g \in C} \sum_{h \in S_{n}} \rho(h)(g, g h)=\sum_{g \in C} \sum_{h^{\prime} \in S_{n}} \rho\left(g^{-1} h^{\prime} g\right)\left(g, h^{\prime} g\right)= \\
\sum_{g \in C} \sum_{h^{\prime} \in S_{n}} \rho\left(h^{\prime}\right)\left(g, h^{\prime} g\right)=\frac{n !}{\rho\left(1_{n}\right)} \Delta_{2}\left(e_{\rho}\right) \cdot \Delta_{1}(\underline{C})
\end{gathered}
$$

whence the required statement follows.

\footnotetext{
${ }^{4}$ Although this statement is a direct consequence of (7.11), [1], we give here a direct proof to make the text self-contained.
} 
Below given three irreducible characters $\rho, \mu, \nu \in \operatorname{Irr}\left(S_{n}\right)$ we set

$$
q(\rho, \mu, \nu)=\frac{\rho\left(2_{n}\right)}{\rho\left(1_{n}\right)}+\frac{\mu\left(2_{n}\right)}{\mu\left(1_{n}\right)}+\frac{\nu\left(2_{n}\right)}{\nu\left(1_{n}\right)}
$$

where $2_{n}=(1,2)$ is the transposition in $S_{n}$.

Theorem 5.2. Let $V=V_{n, 1}$ and $\mathcal{A}=\mathcal{A}_{n}$. Then

$$
V \cap \mathcal{A}=\bigoplus_{\pi: e_{\pi} \in V} \mathcal{A}_{\pi}
$$

Moreover, $e_{\pi}=e_{\rho, \mu, \nu} \in V$ if and only if $q(\rho, \mu, \nu)=-1$.

Proof. Set $\zeta=\zeta_{n, 1}$ and $T=T_{n}$. Then by (15) we have

$$
\zeta=\left(\begin{array}{l}
n \\
2
\end{array}\right) \underline{1}_{n}+\Delta_{1}(\underline{T})+\Delta_{2}(\underline{T})+\Delta_{3}(\underline{T}) .
$$

By Lemma 4.4 given a character $\pi \in \operatorname{Irr}(\mathcal{A})$ there exist characters $\rho, \mu, \nu \in \operatorname{Irr}\left(S_{n}\right)$ such that $e_{\pi}=\Delta_{1}\left(e_{\rho}\right) \cdot \Delta_{2}\left(e_{\mu}\right) \cdot \Delta_{3}\left(e_{\nu}\right)$. Now by Lemma 5.1 we have

$$
\begin{gathered}
\zeta \cdot e_{\pi}=\left(\left(\begin{array}{c}
n \\
2
\end{array}\right) \underline{1}_{n}+\Delta_{1}(\underline{T})+\Delta_{2}(\underline{T})+\Delta_{3}(\underline{T})\right) \cdot \Delta_{1}\left(e_{\rho}\right) \cdot \Delta_{2}\left(e_{\mu}\right) \cdot \Delta_{3}\left(e_{\nu}\right)= \\
\left(\begin{array}{c}
n \\
2
\end{array}\right) e_{\pi}+\Delta_{1}\left(\underline{T} e_{\rho}\right) \cdot \Delta_{2}\left(e_{\mu}\right) \cdot \Delta_{3}\left(e_{\nu}\right)+\Delta_{1}\left(e_{\rho}\right) \cdot \Delta_{2}\left(\underline{T} e_{\mu}\right) \cdot \Delta_{3}\left(e_{\nu}\right)+\Delta_{1}\left(e_{\rho}\right) \cdot \Delta_{2}\left(e_{\mu}\right) \cdot \Delta_{3}\left(\underline{T} e_{\nu}\right)= \\
\left(\begin{array}{l}
n \\
2
\end{array}\right) e_{\pi}+\frac{|T| \rho\left(2_{n}\right)}{\rho\left(1_{n}\right)} e_{\pi}+\frac{|T| \mu\left(2_{n}\right)}{\mu\left(1_{n}\right)} e_{\pi}+\frac{|T| \nu\left(2_{n}\right)}{\nu\left(1_{n}\right)} e_{\pi}=\left(\begin{array}{c}
n \\
2
\end{array}\right)(1+q(\rho, \mu, \nu)) e_{\pi} .
\end{gathered}
$$

This proves the second statement, and by Theorem 4.6 also the first one.

As an immediate consequence of Theorem 5.2 and Lemma 4.4 we obtain the following statement.

Corollary 5.3. The linear space $V_{n, 1}$ is generated as a right ideal of the algebra $\mathbb{Q} S_{n}^{2}$ by the set of all elements $\Delta_{1}\left(e_{\rho}\right) \cdot \Delta_{2}\left(e_{\mu}\right) \cdot \Delta_{3}\left(e_{\nu}\right)$ where $\rho, \mu, \nu \in \operatorname{Irr}\left(S_{n}\right)$ are such that $k_{\mu, \nu}^{\rho} \neq 0$ and $q(\rho, \mu, \nu)=-1$.

Example: The symmetric group $S_{4}$ has 5 irreducible representations; denote them by $\chi_{i}, i=1, \ldots, 5$. The values $\chi_{i}\left(2_{n}\right)$ and $\chi_{i}\left(1_{n}\right)$ for all $i$ are given in the following table (here and below we use tables from [11]):

\begin{tabular}{|c|r|r|r|r|r|}
\hline$i$ & 1 & 2 & 3 & 4 & 5 \\
\hline$\chi_{i}\left(1_{n}\right)$ & 1 & 3 & 2 & 3 & 1 \\
\hline$\chi_{i}\left(2_{n}\right)$ & 1 & 1 & 0 & -1 & -1 \\
\hline $\boldsymbol{\lambda}_{\chi_{i}}$ & {$[4]$} & {$[3,1]$} & {$[2,2]$} & {$[2,1,1]$} & {$\left[1^{4}\right]$} \\
\hline
\end{tabular}

A direct check shows that $q\left(\chi_{i}, \chi_{j}, \chi_{k}\right)=-1$ for $1 \leq i \leq j \leq k \leq 5$ only if $(i, j, k)$ is one of the following triples: $(1,5,5),(3,3,5),(2,4,5)$ and $(4,4,4)$. In all these cases $k_{\chi_{i}, \chi_{j}}^{\chi_{k}}=1$. Thus

$$
\begin{aligned}
V_{4,1}= & V_{1,5,5} \oplus V_{5,1,5} \oplus V_{5,5,1} \oplus \\
& V_{3,3,5} \oplus V_{3,5,3} \oplus V_{5,3,3} \oplus \\
& V_{2,4,5} \oplus V_{2,5,4} \oplus V_{4,2,5} \oplus V_{4,5,2} \oplus V_{5,2,4} \oplus V_{5,4,2} \oplus \\
& V_{4,4,4}
\end{aligned}
$$

where $V_{i, j, k}$ is the right ideal $e_{\chi_{i}, \chi_{j}, \chi_{k}} \mathbb{Q}\left(S_{4} \times S_{4}\right)$. The dimensions of the ideals in the first row are 1 , in the second -4 , in the third -9 and in the fourth -27 . 
Let $\rho \in \operatorname{Irr}\left(S_{n}\right)$ and $\boldsymbol{\lambda}=\boldsymbol{\lambda}_{\rho}$. Then (see the remark in the proof of Theorem 3.5 of $[21])$

$$
\frac{\rho\left(2_{n}\right)}{\rho\left(1_{n}\right)}=\frac{1}{\left(\begin{array}{c}
n \\
2
\end{array}\right)} \sum_{i}\left(\left(\begin{array}{c}
\boldsymbol{\lambda}_{i} \\
2
\end{array}\right)-\left(\begin{array}{c}
\boldsymbol{\lambda}_{i}^{\prime} \\
2
\end{array}\right)\right)
$$

where $\boldsymbol{\lambda}_{i}\left(\right.$ resp. $\left.\boldsymbol{\lambda}_{i}^{\prime}\right)$ is the size of the $i$ th row (resp. the $i$ th column) of $\boldsymbol{\lambda}$. The negative part of the sum in the right-hand side of this equality is more or equal than the ratio $-\boldsymbol{\lambda}_{1}^{\prime} / n$ : this bound is obtained by maximizing $\sum_{i}\left(\boldsymbol{\lambda}_{i}^{\prime}\right)^{2}$ provided that $\boldsymbol{\lambda}_{1}^{\prime} \geq \boldsymbol{\lambda}_{2}^{\prime} \geq \cdots \geq 0$ and $\sum_{i} \boldsymbol{\lambda}_{i}^{\prime}=n$. Applying this bound to each summand of $q(\rho, \mu, \nu)$ we come to the following statement.

Lemma 5.4. Given $\rho, \mu, \nu \in \operatorname{Irr}\left(S_{n}\right)$ we have $q(\rho, \mu, \nu)=-1$ only if at least one of the diagrams $\boldsymbol{\lambda}_{\rho}, \boldsymbol{\lambda}_{\mu}, \boldsymbol{\lambda}_{\nu}$ has at least $\lfloor n / 3\rfloor$ rows.

It is not so difficult to construct infinite families of triples $\rho, \mu, \nu \in \operatorname{Irr}\left(S_{n}\right)$ for which $q(\rho, \mu, \nu)=-1$. For example a straightforward computation by formula (22) shows that if all the diagrams $\boldsymbol{\lambda}_{\rho}, \boldsymbol{\lambda}_{\mu}, \boldsymbol{\lambda}_{\nu}$ are hooks (resp. rectangles), then an infinite family is defined by the condition $r(\rho)+r(\mu)+r(\nu)=2 n+1$ (resp. the conditions $r(\rho)=r(\mu)=n / 2$ and $r(\nu)(r(\nu)-3)=n)$. However, even in these rather simple cases almost nothing is known on the Kronecker coefficients $k_{\rho, \mu}^{\nu}$ (see [3]).

We complete the section by giving an explicit construction of a family of cubic determinants of defect 1 . Take an arbitrary partition $[n]=I \cup J \cup K$, and denote by $\lambda_{I}, \lambda_{J}$ and $\lambda_{K}$ the partitions of $[n]$ into $|I|+1,|J|+1$ and $|K|+1$ classes containing as a class respectively the complements to the sets $I, J$ and $K$. The following lemma shows that the cubic determinant corresponding to the element

$$
D(I, J, K)=\Delta_{1}\left(\chi_{I}\right) \cdot \Delta_{2}\left(\chi_{J}\right) \cdot \Delta_{3}\left(\chi_{K}\right)
$$

with

$$
\chi_{I}=\sum_{g \in S_{\lambda_{I}}}(-1)^{\operatorname{sgn}(g)} g, \quad \chi_{J}=\sum_{g \in S_{\lambda_{J}}}(-1)^{\operatorname{sgn}(g)} g, \quad \chi_{K}=\sum_{g \in S_{\lambda_{K}}}(-1)^{\operatorname{sgn}(g)} g,
$$

is of defect 1 .

Lemma 5.5. For a partition $[n]=I \cup J \cup K$ set $\chi=D(I, J, K)$. Then $\chi \neq 0$ and

(1) $\chi \in V_{n, 1}$,

(2) $\chi^{\lambda_{1}, \lambda_{2}, \lambda_{3}}=0$ for all $\lambda_{1}, \lambda_{2}, \lambda_{3} \in \Lambda(n, r)$ with $r<2 n / 3$.

Proof. It is easily seen that the coefficient of the element $\chi$ at the identity is equal to 1 . Therefore $\chi \neq 0$. Next, by the definition of $V_{n, 1}$ to prove statement (1) it suffices to check that given a transposition $t=(i, j) \in S_{n}$ we have

$$
\Delta_{2}(\underline{T}) \cdot \Delta_{3}(\underline{T}) \cdot \chi=0
$$

where $T=\left\{1_{n}, t\right\}$. Suppose first that $\{i, j\} \not \subset J \cup K$, say $i$ does not belong to $J \cup K$. Then given a permutation $f \in S_{\lambda_{I}}$ the element $j$ is not contained in one of the sets $J^{f^{-1}}$ or $K^{f^{-1}}$. In the former case, $\{i, j\} \cap J=\emptyset$, and hence $(f+t f) \chi_{J}=0$, whereas in the latter case $\{i, j\} \cap K=\emptyset$, and hence $(f+t f) \chi_{K}=0$. Thus in any case

$$
\left.\Delta_{2}(\underline{T}) \cdot \Delta_{3}(\underline{T}) \cdot \Delta_{1}(f) \cdot \Delta_{2}\left(\chi_{J}\right) \cdot \Delta_{3}\left(\chi_{K}\right)=\Delta_{2}\left((f+t f) \chi_{J}\right) \cdot \Delta_{3}(f+t f) \chi_{K}\right)=0
$$


for all $f \in S_{\lambda_{I}}$, and we are done. To complete the proof of (1) let us assume that $\{i, j\} \subset J \cup K$. Then by a direct computation we obtain

$$
\Delta_{2}(\underline{T}) \cdot \Delta_{3}(\underline{T}) \cdot \chi_{I}=\chi^{\prime} \cdot \Delta_{1}\left(\underline{T} \cdot \chi_{I}\right)=0,
$$

because of $\underline{T} \cdot \chi_{I}=0$ where $\chi^{\prime}=\left(1_{n}, 1_{n}\right)+\left(t, 1_{n}\right)$.

To prove statement (2) suppose that $\lambda_{1}, \lambda_{2}, \lambda_{3} \in \Lambda(n, r)$ are partitions such that $\chi^{\lambda_{1}, \lambda_{2}, \lambda_{3}} \neq 0$. It is easily seen that

$$
\chi^{\lambda_{1}, \lambda_{2}, \lambda_{3}}=\Delta_{1}\left(\underline{S}_{\lambda_{1}} \chi_{I}\right) \cdot \Delta_{2}\left(\chi_{J} \underline{S}_{\lambda_{2}}\right) \cdot \Delta_{3}\left(\chi_{K} \underline{S}_{\lambda_{3}}\right),
$$

and hence

$$
\underline{S}_{\lambda_{1}} \cdot \chi_{I} \neq 0, \quad \chi_{J} \cdot \underline{S}_{\lambda_{2}} \neq 0, \quad \chi_{K} \cdot \underline{S}_{\lambda_{3}} \neq 0 .
$$

On the other hand, let a transposition $t$ and the set $T$ be as above. Then obviously $\underline{T} \cdot \chi_{I}=\chi_{I} \cdot \underline{T}=0$ whenever $\{i, j\} \cap I=\emptyset$. Since in the latter case $S_{\lambda_{1}}=S_{\lambda_{1}} T$, we conclude by (24) that at least one of any two distinct elements $i, j$ from the same class of the partition $\lambda_{1}$ belongs to the set $I$. It follows that a class of $\lambda_{1}$ of size $a$ intersects the set $I$ in at least $a-1$ elements. Therefore $r\left(\lambda_{1}\right) \geq n-|I|$. Similarly, one can prove that $r\left(\lambda_{2}\right) \geq n-|J|$ and $r\left(\lambda_{3}\right) \geq n-|K|$. This implies that

$$
3 r=r\left(\lambda_{1}\right)+r\left(\lambda_{2}\right)+r\left(\lambda_{3}\right) \geq 3 n-(|I|+|J|+|K|)=2 n .
$$

Thus $r \geq 2 n / 3$ as required.

\section{Applications to the Border RANK of a CUBIC tensor}

In this section we return to the questions discussed in the introduction. The following metascheme (based on Lemmas 2.4, 3.1 and 3.3) provides a tool to prove that given a positive integer $r$ the border $\operatorname{rank} \underline{\operatorname{rk}}(A)$ of a cubic $n \times n \times n$ tensor $A$ is at least $r$ :

- choose a positive integer $N \geq n$,

- find $\chi \in V_{N, N-r}$ such that $\chi^{\lambda_{1}, \lambda_{2}, \lambda_{3}} \neq 0$ for some $\lambda_{1}, \lambda_{2}, \lambda_{3} \in \Lambda(N, n)$,

- set $P=\left(F_{N}^{-1}(\chi)\right)^{\lambda_{1}, \lambda_{2}, \lambda_{3}}$ (depolarization),

- if $P(A) \neq 0$, then $\underline{\mathrm{rk}}(A) \geq r+1$.

Let us apply this metascheme to the two following concrete examples. The first one shows, in particular, that the bound from the second statement of Lemma 5.5 is attained, whereas the second one gives a lower bound for the border rank of the matrix multiplication tensor.

Example 1. Given a positive odd integer $n=2 m+1$ we define an $n \times n \times n$ zero-one cubic tensor $A=A_{n}$ such that $A_{i, j, k}=1$ if and only if $(i, j, k) \in Q_{n}$ where

$$
\begin{array}{rlrl}
Q_{n}= & \{(n, i, i): & & 1 \leq i \leq m\} \cup \\
& \{(j, n, j+m): & 1 \leq j \leq m\} \cup \\
& \{(k, k, n): & & m<k<n\} .
\end{array}
$$

We claim that

$$
\underline{\mathrm{rk}}\left(A_{n}\right)=\mathrm{rk}\left(A_{n}\right)=3 m=\frac{3(n-1)}{2} .
$$

Indeed, according to our metascheme take $r=3 m-1$ and $N=3 m$. Then by statement (1) of Lemma 5.5 (with $n=N$ ) the linear space $V_{N, N-r}=V_{N, 1}$ contains the nonzero element $\chi=D(I, J, K)$ where

$$
I=\{1, \ldots, m\}, \quad J=\{m+1, \ldots, 2 m\}, \quad K=\{2 m+1, \ldots, 3 m\} .
$$


For $i=1,2,3$ set $\lambda_{i}$ to be the partition in $\Lambda(3 m, 2 m+1)$ of the shape $\left[m, 1^{2 m}\right]$ the unique nonsingleton class of which coincides with the set $I, J$ and $K$ respectively. Then to prove that $\underline{\mathrm{rk}}(A) \geq r+1$ it suffices to verify that $P(A) \neq 0$ where $P(X)$ is the $\left(\lambda_{1}, \lambda_{2}, \lambda_{3}\right)$-contraction of the matching polynomial $F_{N}^{-1}(\chi)$. For this purpose choose the functions $\lambda_{i}, i=1,2,3$ as follows

$$
i^{\lambda_{1}}=\left\{\begin{array}{ll}
n, & \text { if } i \in I, \\
i-m, & \text { if } i \in J \cup K,
\end{array}, \quad k^{\lambda_{3}}= \begin{cases}k, & \text { if } k \in I \cup J, \\
n, & \text { if } k \in K,\end{cases}\right.
$$

and

$$
j^{\lambda_{2}}= \begin{cases}j, & \text { if } j \in I, \\ n, & \text { if } j \in J, \\ j-2 m, & \text { if } j \in K,\end{cases}
$$

Due to $(3)$ and the definition of the $\left(\lambda_{1}, \lambda_{2}, \lambda_{3}\right)$-contraction, the polynomial $P(X)$ can be written in the form

$$
P(X)=\sum_{g \in S_{N}^{2}} \chi(g) M_{g}^{\lambda_{1}, \lambda_{2}, \lambda_{3}}(X),
$$

where $\chi(g) \in\{0, \pm 1\}$ is the coefficient of $\chi$ at $g$. It immediately follows from the definitions of the tensor $A$ and the functions $\lambda_{a}$ that $M_{\left(g_{2}, g_{3}\right)}^{\lambda_{1}, \lambda_{2}, \lambda_{3}}(A) \neq 0$ only if the permutations $g_{2}$ and $g_{3}$ leave the sets $I, J, K$ fixed (as sets) and

$$
g_{2}^{I}=g_{3}^{I}, \quad g_{3}^{J}=1_{J}, \quad g_{2}^{K}=1_{K}
$$

where $1_{J}$ and $1_{K}$ are the identical permutations of $J$ and $K$ respectively. Denote the set of all such pairs $g=\left(g_{2}, g_{3}\right)$ by $H \subseteq S_{N}^{2}$. Then the latter conditions imply that (a) the coefficient $\chi(g)$ is nonzero and does not depend on $g \in H$ (see $(23)$ ), and (b) the monomial $M_{g}^{\lambda_{1}, \lambda_{2}, \lambda_{3}}(X)$ does not depend on $g \in H$, and hence $M_{g}^{\lambda_{1}, \lambda_{2}, \lambda_{3}}(A)=1$. Therefore

$$
P(A)=\sum_{g \in H} \chi(g) M_{g}^{\lambda_{1}, \lambda_{2}, \lambda_{3}}(A)=\chi\left(g_{0}\right)|H| \neq 0
$$

where $g_{0}$ is an arbitrary element from $H$. According to our metascheme this means that $\underline{\mathrm{rk}}(A) \geq 3 \mathrm{~m}$. The converse inequality holds because $\underline{\mathrm{rk}}(A) \leq \mathrm{rk}(A)$, and $\mathrm{rk}(A)$ does not exceed the number of nonzero entries of $A$ that is $\left|Q_{n}\right|=3 \mathrm{~m}$.

Example 2. Set $M_{n}$ to be the structure tensor of $n \times n$-matrix multiplication. As it was mentioned in the introduction $\underline{\mathrm{rk}}\left(M_{n}\right) \geq(2-\epsilon) \cdot n^{2}$ [17]. At present we can not improve this bound, but we can easily apply our metascheme to obtain the lower bound

$$
\underline{\mathrm{rk}}\left(M_{n}\right) \geq \frac{5}{4} n^{2}
$$

Without loss of generality we can assume that $n=2 m$ is even. Set $r=5 m^{2}-1$ and $N=5 m^{2}$. By statement (1) of Lemma 5.5 (with $n=N$ ) the linear space $V_{N, N-r}=V_{N, 1}$ contains the nonzero element $\chi=D(I, J, K)$ where

$$
I=\left\{1, \ldots, m^{2}\right\}, \quad J=\left\{m^{2}+1, \ldots, 3 m^{2}\right\}, \quad K=\left\{3 m^{2}+1, \ldots, 5 m^{2}\right\} .
$$

Now, to prove inequality (27) it suffices to find some partitions $\lambda_{1}, \lambda_{2}$ and $\lambda_{3}$ in the set $\Lambda\left(5 m^{2}, 4 m^{2}\right)$ such that $P(M) \neq 0$ where $M=M_{n}$ and $P(X)$ is the $\left(\lambda_{1}, \lambda_{2}, \lambda_{3}\right)$-contraction of the matching polynomial $F_{N}^{-1}(\chi)$. In fact, it is enough for our purposes that $\lambda_{i} \in \Lambda\left(5 m^{2}, n_{i}^{\prime}\right)$ for some $n_{i}^{\prime} \in\left[4 m^{2}\right]$ (in this case not all 
variables $X_{i j k}$ occur in the polynomial $\left.P(X)\right)$. To construct the partitions choose arbitrarily three bijections:

$$
\begin{array}{ll}
g_{1}: & {\left[4 m^{2}\right] \rightarrow[2 m] \times[2 m],} \\
g_{2}: & {\left[3 m^{2}\right] \rightarrow[2 m] \times[m] \cup[m] \times(m, 2 m],} \\
g_{3}: & {\left[3 m^{2}\right] \rightarrow[2 m] \times(m, 2 m] \cup(m, 2 m] \times[m],}
\end{array}
$$

and define three maps $\lambda_{i}:\left[5 m^{2}\right] \rightarrow[2 m] \times[2 m], i=1,2,3$, such that

$$
\begin{aligned}
& i^{\lambda_{1}}= \begin{cases}\left(\left\lceil\frac{i}{m}\right\rceil,\left\lceil\frac{i}{m}\right\rceil\right), & \text { if } i \in I, \\
i^{g_{1}}, & \text { if } i \in J \cup K,\end{cases} \\
& j^{\lambda_{2}}= \begin{cases}\left(\left\lceil\frac{j-m^{2}}{m}\right\rceil,\left\lceil\frac{j-m^{2}}{m}\right\rceil\right), & \text { if } j \in J, \\
j^{g_{2}}, & \text { if } j \in I \cup K,\end{cases} \\
& k^{\lambda_{3}}= \begin{cases}\left(\left\lceil\frac{k-3 m^{2}}{m}\right\rceil,\left\lceil\frac{k-3 m^{2}}{m}\right\rceil\right), & \text { if } k \in K, \\
k^{g_{3}}, & \text { if } k \in I \cup J,\end{cases}
\end{aligned}
$$

From the definition it immediately follows that

$$
\begin{aligned}
\lambda_{1} \in \Lambda\left(5 m^{2}, 4 m^{2}\right) & \text { and } & & {\left[\lambda_{1}\right]=\left[(m+1)^{m}, 1^{4 m^{2}-m}\right], } \\
\lambda_{2} \in \Lambda\left(5 m^{2}, 3 m^{2}+m\right) & \text { and } & & {\left[\lambda_{2}\right]=\left[(m+1)^{m}, m^{m}, 1^{3 m^{2}-m}\right], } \\
\lambda_{3} \in \Lambda\left(5 m^{2}, 3 m^{2}+m\right) & \text { and } & & {\left[\lambda_{3}\right]=\left[m^{2 m}, 1^{3 m^{2}}\right] . }
\end{aligned}
$$

Due to (3) and the definition of a contraction the polynomial $P(X)$ can be written in the form (26). It immediately follows from the definitions of the tensor $M$ that given $g \in S_{N} \times S_{N}$, we have $M_{g}^{\lambda_{1}, \lambda_{2}, \lambda_{3}}(M) \neq 0$ only if $\left(i^{\lambda_{1}}, i^{\lambda_{2} g_{2}}, i^{\lambda_{3} g_{3}}\right)=(u v, v w, w u)$ for some elements $u, v, w \in[2 m]$ where $u v=(u, v), v w=(v, w)$ and $w u=(w, u)$. In this case the above triple obviously belongs to one of the following sets:

$$
\begin{aligned}
\{(u v, v u, u u) & : u v \in[m] \times[2 m]\}, \\
\{(u v, v v, v u) & : u v \in(m, 2 m] \times[2 m]\}, \\
\{(u u, u w, w u) & : u w \in[m] \times(m, 2 m]\} .
\end{aligned}
$$

Now, denote by $H$ the set of all $g=\left(g_{2}, g_{3}\right) \in S_{N}^{2}$ for which $M_{g}^{\lambda_{1}, \lambda_{2}, \lambda_{3}}(M) \neq 0$. Then the argument as in Example 1 shows that (a) the coefficient $\chi(g)$ is nonzero and does not depend on $g \in H$, and (b) the monomial $M_{g}^{\lambda_{1}, \lambda_{2}, \lambda_{3}}(X)$ does not depend on the element $g \in H$. Therefore $P(M) \neq 0$, and hence $\underline{\text { rk }}(M) \geq 5 \mathrm{~m}^{2}$.

Remark. The above proof shows that the structure tensor of the $2 m \times 2 m$ matrix multiplication contains a $4 m^{2} \times\left(3 m^{2}+m\right) \times\left(3 m^{2}+m\right)$ subtensor the border rank of which is at least $5 \mathrm{~m}^{2}$.

Acknowledgements. This paper was written during the authors visiting Max Planck Institut für Mathematik in Bonn. We would like to express our gratitude to the Institute for hospitality and excellent working conditions. The first author is grateful to Labex CEMPI (ANR-11-LABX-0007-01). Also the authors are thankful to the anonymous referees whose valuable remarks helped to improve the exposition.

\section{REFERENCES}

[1] E. Bannai, T. Ito. Algebraic Combinatorics I: Association Schemes. Benjamin-Cummings, Menlo Park (1984).

[2] M. Bläser, A $\frac{5}{2} n^{2}$-lower bound for the rank of $n \times n$-matrix multiplication over arbitrary fields, IEEE Symp. Found. Comput. Sci. , New-York, 1999, 45-50.

[3] P. Bürgisser, M. Christandl, C. Ikenmeyer, Nonvanishing of Kronecker coefficients for rectangular shapes, Adv. Math., 227 (2011), 2082-2091. 
[4] P. Bürgisser, M. Clausen, A. Shokrollahi, Algebraic complexity theory, Springer, Berlin, 1997 (with the collaboration of T. Lickteig).

[5] P. Bürgisser, C. Ikenmeyer, Geometric Complexity Theory and Tensor Rank, ACM Symp. Th. Comput., New-York, 2011, 509-518.

[6] P. Bürgisser, C. Ikenmeyer, Explicit Lower Bounds via Geometric Complexity Theory, ACM Symp. Th. Comput., New-York, 2013, 141-150.

[7] J. Buczynski, J. M. Landsberg, Ranks of tensors and a generalization of secant varieties, Lin. Alg. Appl., 438 (2013), 668-689.

[8] D. Grigoriev, Lower bounds in algebraic complexity, J. Soviet Math., 29 (1985), 1388-1425.

[9] D. Grigoriev, N. Vorobjov, Bounds on numbers of vectors of multiplicities for polynomials which are easy to compute, Proc. of the 2000 International Symposium on Symbolic and Algebraic Computation (St. Andrews), 137-145, ACM, New York, 2000.

[10] I. M. Isaacs, Character theory of finite groups, Academic Press, 1976.

[11] G. D. James, A. Kerber, The Representation Theory of the Symmetric Group, Encyclopedia of Mathematics, vol. 16, 1981.

[12] J. M. Landsberg, Geometry and the complexity of matrix multiplication, Bull. AMS, 46 (2008), 247-284.

[13] J. M. Landsberg, Tensors: Geometry and Applications, AMS, 2011.

[14] J. M. Landsberg, L. Manivel, On the ideals of secant varietes of Segre varietes, Found. Comput. Math., 4 (2004), 397-422.

[15] J. M. Landsberg, L. Manivel, Generalizations of Strassen's equations for secant varieties of Segre varietes, Commun. Algebra, 36 (2008), 1-18.

[16] J. M. Landsberg, G. Ottaviani, Equations for secant varieties of Veronese and other varieties, Annali di Matematica Pura e Applicata, 192 (2013), 569-606.

[17] J. M. Landsberg, G. Ottaviani, New lower bounds for the border rank of matrix multiplication, arXiv:1112.6007v1 [math.AG], 2011, 1-11.

[18] J. M. Landsberg, Z. Teitler, On the ranks and border ranks of symmetric tensors, Found. Comput. Math., 10 (2010), 339-366.

[19] T. Lickteig, A note on border rank, Inform. Process. Lett., 18 (1984), no. 3, 173-178.

[20] H. Nagao, Y. Tsushima, Representations of finite groups, Academic Press, 1989.

[21] Y. Roichman, Upper bound on the characters of the symmetric groups, Invent. Math., 125 (1996), 451-485.

[22] V. Strassen, Vermeidung von Divisionen, J. Reine Angew. Math., 264 (1973), 184-202.

[23] V. Strassen, Rank and optimal computation of generic tensors, Linear Alg. Appl., 52/53 (1983), 645-685.

[24] V. Strassen, Relative bilinear complexity and matrix multiplication, J. Reine Angew. Math., $\mathbf{3 7 5 / 3 7 6}$ (1987), 406-443.

[25] V. Strassen, Algebraic complexity theory, in: Handbook of Theor. Comput. Sci., vol. A, 1990, Elsevier, Amsterdam, 633-672.

[26] H. Wielandt, Finite permutation groups, Academic Press, New York - London, 1964.

CNRS, Mathématiques, Université de Lille, France

E-mail address: dmitry.grigoryev@math.univ-lille1.fr

Netanya Academic College, Netanya, Israel

E-mail address: muzy@netanya.ac.il

Steklov Institute of Mathematics at St. Petersburg, Russia

E-mail address: inp@pdmi.ras.ru 